Methodology article

\title{
Detecting purely epistatic multi-locus interactions by an omnibus permutation test on ensembles of two-locus analyses Waranyu Wongseree ${ }^{1}$, Anunchai Assawamakin ${ }^{2}$, Theera Piroonratana ${ }^{1}$, Saravudh Sinsomros ${ }^{1}$, Chanin Limwongse ${ }^{2}$ and Nachol Chaiyaratana ${ }^{* 1,2}$
}

Address: ${ }^{1}$ Department of Electrical Engineering, Faculty of Engineering, King Mongkut's University of Technology North Bangkok, 1518 Piboolsongkram Road, Bangsue, Bangkok 10800, Thailand and 2Division of Molecular Genetics, Department of Research and Development, Faculty of Medicine Siriraj Hospital, Mahidol University, 2 Prannok Road, Bangkoknoi, Bangkok 10700, Thailand

Email: Waranyu Wongseree - waranyu.wongseree@gmail.com; Anunchai Assawamakin - anunchai_ice@yahoo.com;

Theera Piroonratana - theepi@gmail.com; Saravudh Sinsomros - 2saravudh@gmail.com; Chanin Limwongse - siclw@mahidol.ac.th; Nachol Chaiyaratana* - n.chaiyaratana@gmail.com

* Corresponding author

Published: 17 September 2009

BMC Bioinformatics 2009, 10:294 doi:10.1186/1471-2105-10-294
Received: 5 March 2009

Accepted: 17 September 2009

This article is available from: http://www.biomedcentral.com/I47/-2/05/I0/294

(c) 2009 Wongseree et al; licensee BioMed Central Ltd.

This is an Open Access article distributed under the terms of the Creative Commons Attribution License (http://creativecommons.org/licenses/by/2.0), which permits unrestricted use, distribution, and reproduction in any medium, provided the original work is properly cited.

\begin{abstract}
Background: Purely epistatic multi-locus interactions cannot generally be detected via singlelocus analysis in case-control studies of complex diseases. Recently, many two-locus and multilocus analysis techniques have been shown to be promising for the epistasis detection. However, exhaustive multi-locus analysis requires prohibitively large computational efforts when problems involve large-scale or genome-wide data. Furthermore, there is no explicit proof that a combination of multiple two-locus analyses can lead to the correct identification of multi-locus interactions.
\end{abstract}

Results: The proposed 2LOmb algorithm performs an omnibus permutation test on ensembles of two-locus analyses. The algorithm consists of four main steps: two-locus analysis, a permutation test, global $p$-value determination and a progressive search for the best ensemble. 2LOmb is benchmarked against an exhaustive two-locus analysis technique, a set association approach, a correlation-based feature selection (CFS) technique and a tuned ReliefF (TuRF) technique. The simulation results indicate that $2 \mathrm{LOmb}$ produces a low false-positive error. Moreover, $2 \mathrm{LOmb}$ has the best performance in terms of an ability to identify all causative single nucleotide polymorphisms (SNPs) and a low number of output SNPs in purely epistatic two-, three- and four-locus interaction problems. The interaction models constructed from the 2LOmb outputs via a multifactor dimensionality reduction (MDR) method are also included for the confirmation of epistasis detection. 2LOmb is subsequently applied to a type 2 diabetes mellitus (T2D) data set, which is obtained as a part of the UK genome-wide genetic epidemiology study by the Wellcome Trust Case Control Consortium (WTCCC). After primarily screening for SNPs that locate within or near 372 candidate genes and exhibit no marginal single-locus effects, the T2D data set is reduced to 7,065 SNPs from 370 genes. The $2 \mathrm{LOmb}$ search in the reduced T2D data reveals that four intronic SNPs in PGMI (phosphoglucomutase I), two intronic SNPs in LMXIA (LIM homeobox transcription factor I, alpha), two intronic SNPs in PARK2 (Parkinson disease (autosomal recessive, juvenile) 2, parkin) and three intronic SNPs in GYS2 (glycogen synthase 2 (liver)) are associated with the disease. The $2 \mathrm{LOmb}$ result suggests that there is no interaction between each pair of the identified 
genes that can be described by purely epistatic two-locus interaction models. Moreover, there are no interactions between these four genes that can be described by purely epistatic multi-locus interaction models with marginal two-locus effects. The findings provide an alternative explanation for the aetiology of T2D in a UK population.

Conclusion: An omnibus permutation test on ensembles of two-locus analyses can detect purely epistatic multi-locus interactions with marginal two-locus effects. The study also reveals that SNPs from large-scale or genome-wide case-control data which are discarded after single-locus analysis detects no association can still be useful for genetic epidemiology studies.

\section{Background}

Complex diseases cannot generally be explained by Mendelian inheritance [1] because they are influenced by gene-gene and gene-environment interactions. Many common diseases such as asthma, cancer, diabetes, hypertension and obesity are widely accepted and acknowledged to be results of complex interactions between multiple genetic factors [2]. Attempts to identify factors that could be the causes of complex diseases have led to many genome-wide association studies $[3,4]$. Raw results from these attempts produce a large amount of single nucleotide polymorphism (SNP) data from every individual participating in the trials.

For genetic epidemiologists, data sets from genome-wide association studies present many challenges, particularly the correct identification of SNPs that associate with the disease of interest from all available SNPs [5]. This challenge can be treated as a pattern recognition problem which aims to identify an attribute or SNP set that can lead to the correct classification of recruited samples. Heidema et al. [5] and Motsinger et al. [6] have reviewed and identified many machine learning techniques that are suitable to the task. Among many strategies and techniques, the protocol that appears to be most promising for genome-wide association studies involves two main steps: SNP set reduction and classification model construction [7]. From a machine learning viewpoint, attribute selection techniques can be divided into three main categories: filter, wrapper and embedded approaches [8]. In a filter approach, a measure or an index is used to determine the correlation between attributes and classes, e.g. affected and unaffected status in a case-control study. Attributes that are deemed to be important for the classification according to the measure are then selected. The filter approach includes $\chi^{2}$ and odds ratio tests $[9,10]$, omnibus permutation tests [11-13], a correlation-based feature selection technique [14], a ReliefF technique [15] and a tuned ReliefF technique [16]. In a wrapper approach, the significance of an attribute subset is evaluated from the classification performance by a classifier. The capability of the wrapper approach to identify significant attributes thus depends on the chosen classifier and the search algorithm for the identification of the best attribute subset.
Combinatorial [17] and restricted partitioning methods [18], a multifactor dimensionality reduction method [1925] and a polymorphism interaction analysis technique [26] are examples of the wrapper approach. An embedded approach concentrates on informative attributes during the construction of a classification model. Examples of the embedded approach include a genetic algorithm with Boolean algebra [27], genetic programming based decision trees [28,29], random forests [30-32] and evolutionary neural networks $[33,34]$. Based on this categorisation, classification models are not direct outputs from filterbased techniques. On the other hand, classification models are readily prepared as outputs from the wrapper and embedded approaches. In other words, the last two approaches can also be regarded as classification model construction techniques.

The success of the two-step pattern recognition approach relies heavily on the attribute selection step [14]. In casecontrol studies, epistatic effects play a vital role in establishing the difficulty level of SNP screening problems $[35,36]$. Epistasis in the simplest form can be represented by disease models that require genotype inputs from two interacting SNPs $[37,38]$. Many attempts have been made to produce consistent definitions and categorisation of different types of epistasis models [2,35,39-41]. According to Musani et al. [2], a pure epistasis model [42] is difficult because each SNP exhibits no marginal single-locus effect in the model. As a result, it is impossible to detect the pure epistasis by univariate statistical tests. Examples of complex diseases that case-control studies have uncovered putatively pure epistasis include type 2 diabetes mellitus (T2D) [43-46] and metabolic syndrome [47]. Due to the difficulty of screening for each SNP independently, it is suggested that attention should be focused on the analysis of differences between two-locus genotype distribution within case and control groups [40] and multi-locus Bayesian statistical analysis $[48,49]$.

A number of SNP screening and association detection techniques have adopted the two-locus genotype monitoring strategy as their core engines [40,50-52]. The search for interactions can be carried out via either exhaustive analysis [52] or the analysis that can be divided into two 
stages, incorporating single-locus analysis for the prescreening purpose $[40,50,51]$. In the two-stage mode, at least one SNP that involves in the construction of twolocus genotype unit must be a strong candidate for the association explanation, usually verified through univariate statistical tests. Each mode of the two-locus analysis possesses different strengths and weaknesses. The exhaustive analysis has a full capability of detecting pure epistasis but requires larger computational efforts [52]. In contrast, the two-stage analysis is more practical for large-scale data but with some risk of missing possible pure epistasis [50]. More practical usage of both two-locus analysis modes in real case-control studies is required before the feasibility issue can be fully addressed.

Many genetic association studies reveal that various complex diseases are results of putative multi-locus interactions $[11,46,53]$. With the constraints on a computational capability, exhaustive multi-locus analysis in large-scale or genome-wide association studies would be infeasible [52]. On the other hand, single-locus analysis would be unsuitable for the detection of pure epistasis. One possible approach that provides a trade-off between a computational limitation and an epistasis detection capability is to capture a multi-locus interaction by combining multiple results from two-locus analysis. To achieve this, it is necessary to prove that once a multi-locus interaction model is broken down into a combination of two-locus models, all or some of these models remain detectable through two-locus analysis. Although it is hinted in an early work on two-locus analysis [52] that the proposed approach is plausible, explicit experimentation and testing has never been conducted.

In this article, the feasibility of employing an ensemble of two-locus analyses for the multi-locus interaction determination is demonstrated. Specifically, the significance of the two-locus analysis ensemble is assessed by an omnibus permutation test [54]. The proposed method is inspired by a set association approach [11], in which a limited number of sets that contain different numbers of SNPs are explored for possible association. These SNP sets are crucial in the global $p$-value calculation of the selected set via a permutation test and thus the decision to accept or reject the null hypothesis of no association. In other words, SNP set exploration and selection is required to assess the significance of the identified association. This means that the set association approach is equally interested in both SNP set selection and testing for significant association. The primary function of the proposed method is to detect possible association and assess its significance through the exploration of different ensembles of two-locus analyses. Hence, the proposed method is also equally interested in both ensemble selection and testing for significant association.
The proposed method is benchmarked against a simple exhaustive two-locus analysis technique, the set association approach [11], the correlation-based feature selection technique [14] and the tuned ReliefF technique [16]. These filter-based attribute selection techniques are suitable for the benchmark trial since they are capable of detecting association. The case-control classification models constructed from screened SNPs via a multifactor dimensionality reduction method [19] are also provided.

\section{Results and discussion Algorithm}

The proposed algorithm performs an omnibus permutation test on ensembles of two-locus analyses and is referred to as a $2 \mathrm{LOmb}$ technique. The algorithm consists of four steps as illustrated in Figure 1 and can be described as follows.

\section{Two-locus analysis}

Consider a case-control genetic association study with $n_{m}$ SNPs, for each pair of SNPs, a $2 \times 9$ contingency table with rows for disease status and columns for genotype configurations is created. A $\chi^{2}$ test statistic and the corresponding $p$-value can subsequently be computed. With the total of $n_{m}$ SNPs, there are $\left(\begin{array}{c}n_{m} \\ 2\end{array}\right)=n_{m} ! /\left(\left(n_{m}-2\right) ! 2 !\right)$ possible SNP pairs. As a result, the $p$-value from each two-locus analysis must be adjusted by a Bonferroni correction. The Bonferroni-corrected $p$-value from each analysis is the lower value between $\left(\begin{array}{c}n_{m} \\ 2\end{array}\right) \times$ the uncorrected $p$-value and one.

\section{Permutation test}

The $p$-value $p_{0}^{e}$ for the null hypothesis $H_{0}^{e}$ that ensemble $e$-an ensemble of two-locus analyses of interest-is not associated with the disease can be evaluated by a permutation test. To achieve this, a scalar statistic is first computed from a function that combines the Bonferronicorrected $\chi^{2}$ s $p$-values of individual two-locus tests. A suitable combining function must (a) be non-increasing in each $p$-value, (b) attain its maximum value when any $p$ value equals to zero and (c) have a finite critical value that is less than its maximum for any significant level greater than zero [54]. In this study, a Fisher's combining function $\left(-2 \sum_{i} \log \left(p_{i}\right)\right)$ is selected [55]. The $p$-value for the ensemble of two-locus analyses is assessed via a permutation simulation. In each permutation replicate, samples are constructed such that the case/control status of each sample is randomly permuted while the total numbers of 


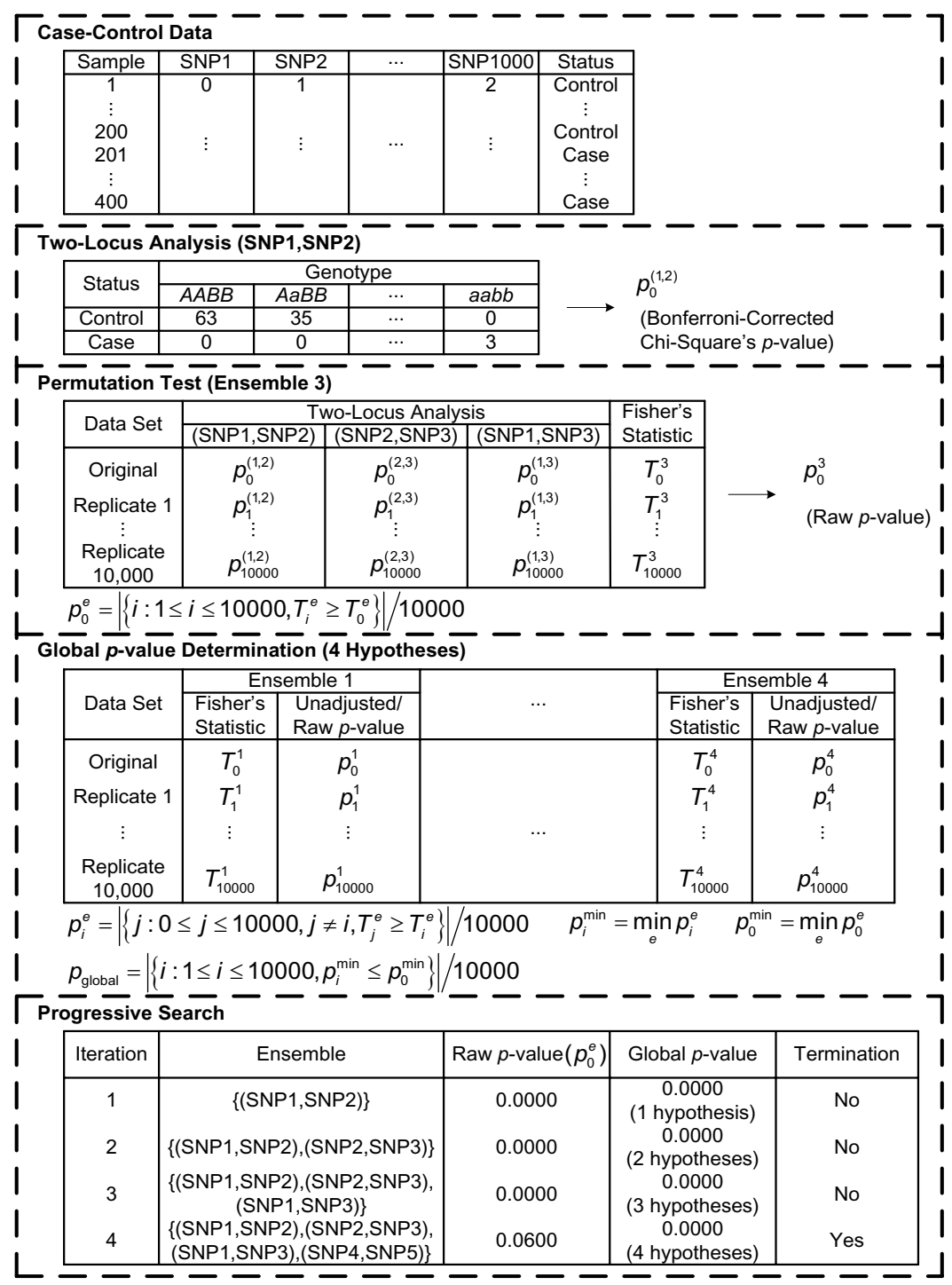

Figure I

Outline of $\mathbf{2 L O m b}$. In this example, the algorithm takes a balanced case-control data set that consists of 400 samples and I,000 SNPs. Each genotype is represented by an integer: 0 denotes a homozygous wild-type genotype, I denotes a heterozygous genotype and 2 denotes a homozygous variant or homozygous mutant genotype. A $\chi^{2}$ contingency table is then constructed for each pair of SNPs in two-locus analysis. This results in the total of $\left(\begin{array}{c}1,000 \\ 2\end{array}\right)=499,500$ two-locus analyses. Thus, the Bonferroni-corrected $\chi^{2}$ 's $p$-value for each two-locus analysis is the lower value between $499,500 \times$ its uncorrected $p$-value and one. In one ensemble, Bonferroni-corrected $\chi^{2}$ s $p$-values from multiple two-locus analyses are combined together via a Fisher's combining function, which in turn provides a Fisher's test statistic result. The raw $p$-value for the ensemble is obtained through a permutation test, which is composed of 10,000 randomised permutation replicates. Since multiple ensembles may be tried during the identification of the best association explanation, a global $p$-value is calculated to account for multiple hypothesis testing. The global $p$-value is estimated through the same permutation test that gives the raw $p$-value for each ensemble. The progressive search for the best association explanation is carried out by incrementally adding a two-SNP unit to the current best ensemble. The condition for search termination is based on both the raw $p$-value for the explored ensemble and the global $p$-value. In this example, the search is terminated after the fourth ensemble is explored due to an increase in the raw $p$ value. Subsequently, the best SNP set for association explanation contains SNPI, SNP2 and SNP3 where the global $p$-value that accounts for testing of four hypotheses is $p<0.000$ I. 
case and control samples remain unchanged. A $\chi^{2}$ contingency table with new entries and a Bonferroni-corrected $p$ value for the two-locus analysis within each permutation replicate are then obtained. This, in turn, leads to a new Fisher's test statistic. Let $T_{i}^{e}$ denote the value of Fisher's test statistic obtained for the $i$ th permutation replicate, $p_{0}^{e}$ is the fraction of permutation replicates with a test statistic greater than or equal to the test statistic obtained from the original case-control data $\left(T_{0}^{e}\right)$. In other words,

$$
p_{0}^{e}=\left|\left\{i: 1 \leq i \leq t, T_{i}^{e} \geq T_{0}^{e}\right\}\right| / t,
$$

where $t$ is the number of permutation replicates which is set to 10,000 in this study and $|\cdot|$ denotes the size of a set.

\section{Global p-value determination}

There are many candidate ensembles of two-locus analyses that can be explored. Let $H_{0}=\bigcap_{1 \leq e \leq E} H_{0}^{e}$ be the global null hypothesis that none of $E$ explored ensembles of two-locus analyses is associated with the disease, the test of the global null hypothesis leads to the global $p$-value and provides the genetic association explanation. In step 2 , the $p$-value $p_{0}^{e}$ for a fixed hypothesis $H_{0}^{e}$ is a raw or unadjusted $p$-value. To account for the correlation among multiple hypotheses that have been tested during the exploration through many candidate ensembles, the testing result of the global null hypothesis depends on $p_{0}^{\min }=\min _{e} p_{0}^{e}$. In other words, the global null hypothesis is rejected if the minimum of the raw $p$-values is sufficiently small. The distribution of $p_{0}^{\min }$ can again be determined by a permutation simulation. However, a nested simulation is unnecessary since the same set of permutation replicates for the $p_{0}^{e}$ determination can be reused in the estimation of the empirical distribution of $p_{0}^{\min }[56]$. This strategy has been successfully implemented in a number of genetic association detection techniques, including a set association approach [11] and a haplotype interaction approach embedded in FAMHAP $[57,58]$. The unadjusted $p$-value for the permutation replicate $i$ of each hypothesis $e$ is thus given by

$$
p_{i}^{e}=\left|\left\{j: 0 \leq j \leq t, j \neq i, T_{j}^{e} \geq T_{i}^{e}\right\}\right| / t .
$$

Let $p_{i}^{\min }=\min _{e} p_{i}^{e}$ be the minimum of unadjusted $p$-values over all explored ensembles of two-locus analyses in the ith permutation replicate, the $p$-value for the global null hypothesis $H_{0}$ is defined by

$$
p_{\text {global }}=\left|\left\{i: 1 \leq i \leq t, p_{i}^{\min } \leq p_{0}^{\min }\right\}\right| / t .
$$

Search for the best ensemble of two-locus analyses

A simple progressive search is used to identify the best ensemble of two-locus analyses. The search begins by locating the best two-SNP unit with the smallest Bonferroni-corrected $\chi^{2}$ s $p$-value from step 1 . A permutation test is then performed for this two-locus analysis, yielding both raw and global $p$-values since only one hypothesis has been explored. Next, the search attempts to combine the existing best two-SNP unit with the two-SNP unit that possesses the next smallest Bonferroni-corrected $\chi^{2}$ s $p$ value from step 1 and does not have a higher permutation $p$-value than the first two-SNP unit. If this new ensemble yields either a higher raw $p$-value or the same raw $p$-value but a higher global $p$-value from a permutation test, the search is terminated and the association is explained by the previously identified two-locus analysis. Otherwise, the best ensemble of two-locus analyses is updated and the process of appending more two-SNP units to the ensemble continues. The progressive search terminates when deterioration in the raw or global $p$-value is detected, or all possible two-locus analyses have been included in the ensemble. It is recalled from step 3 that for the best ensemble containing $E-1<\left(\begin{array}{c}n_{m} \\ 2\end{array}\right)$ two-locus analyses, its global $p$-value is obtained from the evaluation of $E$ hypotheses.

\section{Validity of the algorithm}

A permutation replicate in $2 \mathrm{LOmb}$ is constructed by randomly assigning the case or control status to each sample while maintaining the original proportion of case and control samples. Once the construction of a permutation replicate is finished, the assigned case and control labels remain fixed to the samples. The pattern of case and control labels in each permutation replicate is thus constant and unique. Therefore, the Bonferroni-corrected $\chi^{2} \mathrm{~s} p$ values from any two-SNP units within a permutation replicate are calculated from the same case-control data set. Hence, the combining of these Bonferroni-corrected $\chi^{2}$ s $p$-values via a Fisher's combining function is attainable. The calculation of Fisher's test statistics from all permutation replicates and the original data set leads to the raw or unadjusted $p$-value $p_{0}^{e}$ for the null hypothesis $H_{0}^{e}$ of the 
ensemble $e$ as given in equation 1. Since the same set of permutation replicates is always used during the evaluation of each ensemble, the raw $p$-values for the null hypotheses from all ensembles can be directly compared against one another. Furthermore, the global $p$-value calculation is based on this set of permutation replicates. This is possible because the unadjusted $p$-value for the permutation replicate $i$ of ensemble $e$ or $p_{i}^{e}$ can be calculated in a similar manner to the raw $p$-value $p_{0}^{e}$ as defined in equation 2 . The unadjusted $p$-values for the same permutation replicate but different ensembles can also be directly compared and the subsequent calculation of $p_{i}^{\min }=\min _{e} p_{i}^{e} \quad$ is attainable. With $p_{i}^{\text {min }}$ and $p_{0}^{\min }=\min _{e} p_{0}^{e}$, the $p$-value for the global null hypothesis $H_{0}=\bigcap_{1 \leq e \leq E} H_{0}^{e}$ that incorporates all $E$ explored hypotheses can be determined by equation 3. In summary, only one set of permutation replicates is required for the calculation of both the raw $p$-value for the null hypothesis of every ensemble and the global $p$-value. The $p$-values can be compared in each step of $2 \mathrm{LOmb}$. Consequently, the selection of the best ensemble for association explanation can be carried out via a $p$-value comparison.

\section{Testing with simulated data}

2LOmb is benchmarked against a simple exhaustive twolocus analysis technique, a set association approach (SAA) [11], a correlation-based feature selection (CFS) technique [14] and a tuned ReliefF (TuRF) technique [16] in a simulation trial. The exhaustive two-locus analysis is simply the two-locus analysis procedure from the first step of the $2 \mathrm{LOmb}$ algorithm. An interaction is declared if at least one two-SNP unit with a Bonferroni-corrected $\chi^{2}$ 's $p$-value below 0.05 is detected. The exhaustive two-locus analysis reports all SNPs that meet this detection condition. The simulation covers two main data categories: null data of no significant genetic association and data with causative SNPs which signify pure epistasis. The algorithm performance on the null data provides an indication for the falsepositive error. On the other hand, the algorithm performance on the data with causative SNPs indicates the detection capability. An efficient algorithm should produce an output with a low number of SNPs and a high number of correctly-identified causative SNPs when epistasis is present. Similarly, it should also report that there are no causative SNPs in the null data. These two measures on the number of SNPs in the results are used as the performance indicators.

Each simulated data set contains $1,000,2,000$ or 4,000 SNPs in which either there are no causative SNPs or there is pure epistasis, governed by two, three or four causative SNPs. The allele frequencies of all causative SNPs are 0.5 while the minor allele frequencies of the remaining SNPs are between 0.05 and 0.5 . The data set consists of balanced case-control samples of sizes 400,800 or 1,600. All SNPs in control samples are in Hardy-Weinberg equilibrium (HWE) [59]. The genotype distribution of causative interacting SNPs follows the pure epistasis model by Culverhouse et al. [42], leading to three interesting values of heritability: 0.01, 0.025 and 0.05. Every SNP in each data set exhibits no marginal single-locus effect (Bonferronicorrected $\chi^{2}$ s $p$-value $>0.05$ ). Twenty-five independent data sets for each simulation setting are generated via a genomeSIM package [60]. A paired $t$-test is suitable to assess the significance of results since the same simulated data sets are used during the algorithm benchmarking.

The results from the null data problem are summarised in Figure 2 while the results from the two-, three-and fourlocus interaction problems are shown in Figures 3-4, 5-6 and 7-8, respectively. Clearly, $2 \mathrm{LOmb}$ significantly outperforms other techniques in terms of the low number of output SNPs, the high number of correctly-identified causative SNPs or both in every interaction problem (a paired $t$-test on 675 benchmark results yields a $p$-value < $0.05)$. On the other hand, both $2 \mathrm{LOmb}$ and SAA have the lowest false-positive error when compared to other techniques in the null data problem (a paired $t$-test on 225 benchmark results yields a $p$-value $<0.05$ ). The statistical power analysis also reveals that the benchmark trial with 25 independent data sets for each simulation setting is sufficient for an accurate evaluation of the overall algorithm performance (power $>0.95$ for a Type I error rate of $0.05)$. These results can be further interpreted as follows.

The performance of many existing attribute selection techniques for pattern recognition depends on the level of attribute interactions. A number of techniques, including CFS, appear to function well under a moderate level of interactions. However, the performance of CFS appears to be significantly reduced when the interaction level becomes too high $[14,61]$ because CFS favours an attribute that is strongly correlated with the classification outcome--disease status in this study--while at the same time is not correlated with other attributes. Since the main driving force behind epistasis is the interaction between SNPs, which are themselves attributes, CFS would not intuitively select all causative SNPs. Consequently, the SNP set produced by CFS appears to contain only uncorrelated SNPs. Obviously, a SNP that is a part of the interaction model would occasionally be picked up by CFS but CFS never successfully identifies all causative SNPs in any interaction problems. In addition, CFS reports more erroneous SNPs than other techniques in the null data problem and all three interaction problems due to many SNPs being uncorrelated. 

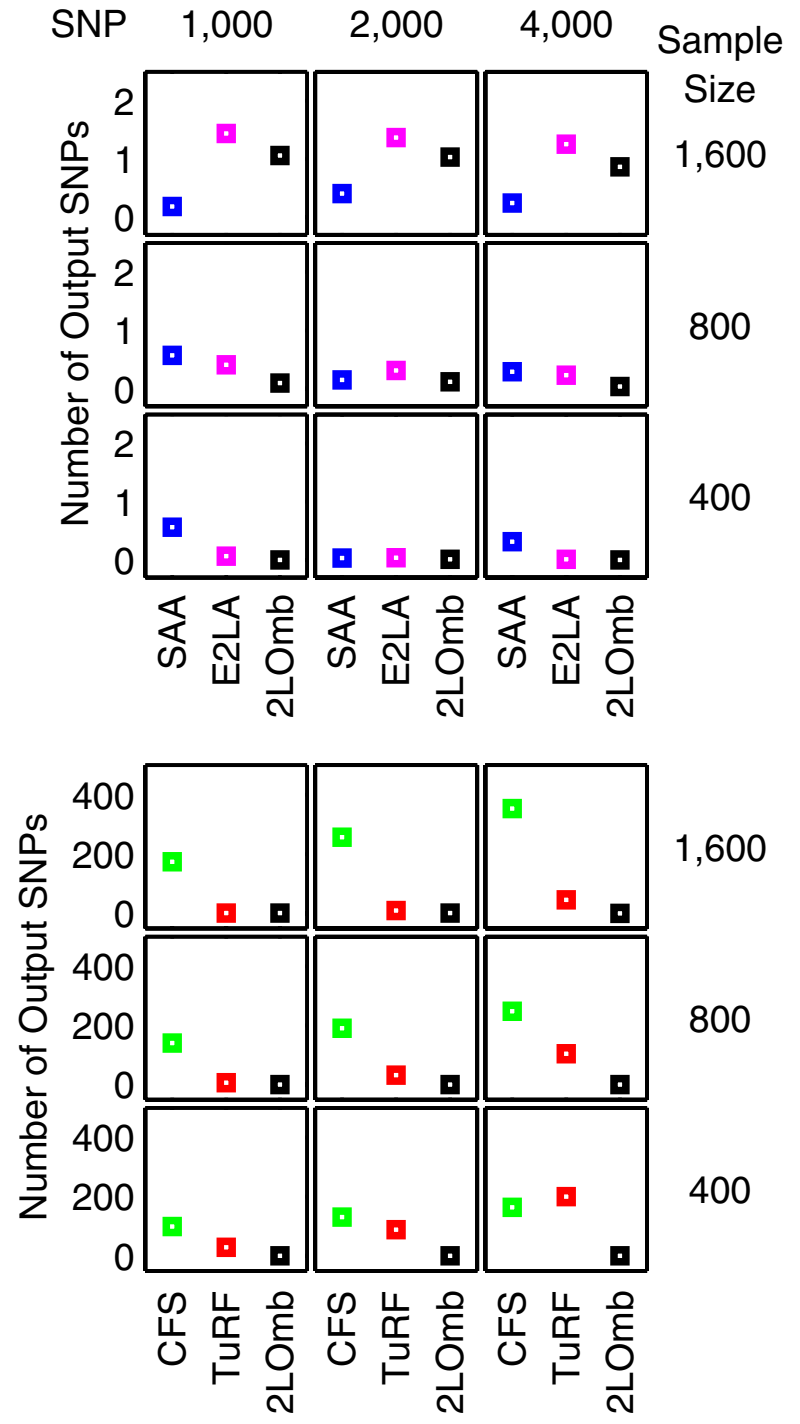

Figure 2

Performance of the exhaustive two-locus analysis, SAA, CFS, TuRF and 2LOmb in the null data problem. The results are averaged over 25 independent simulations. False detection is declared for the exhaustive twolocus analysis, SAA and 2LOmb if the $p$-values used as detection indicators in their results are less than 0.05 . The results from the exhaustive two-locus analysis (E2LA), SAA, CFS, TuRF and 2LOmb are displayed using magenta, blue, green, red and black markers, respectively. In each chart, the horizontal axis represents the detection algorithm while the vertical axis represents the number of output SNPs reported by the algorithm. The top nine charts are displayed using a finer scale than the bottom nine charts.
The benchmarking of attribute selection techniques by Hall and Holmes [14] also reveals that ReliefF [15] is better than CFS in problems with a high level of interactions. Since ReliefF is essentially the core engine of TuRF, the results from this study are in agreement with the early benchmark trial. This finding strengthens the observation that the interaction level of SNPs in pure epistasis models is too high for CFS to handle. Similar to its predecessor, the performance of TuRF still depends on both the number of attributes and sample size. TuRF performs well in the majority of simulation scenarios with $1,000-2,000$ SNPs and 800-1,600 samples. These scenarios are relatively easy since the number of SNPs is small while the sample size is large. However, the size of output SNP set, reported by TuRF from the null data problem and all three interaction problems, increases significantly when the difficulty level rises by either reducing the sample size or increasing the number of SNPs. This implies that when the problem contains a large number of candidate SNPs, the only way to ensure that TuRF reports a proper SNP set is to use a relatively large sample size, making it impractical in real genetic association studies due to many factors including disease prevalence, population size and genotyping cost.

The global $p$-values in most of the SAA results from the null data problem and all three interaction problems exceed 0.05 , showing that SAA reports a low false-positive result in the null data problem. Nonetheless, SAA remains unsuitable for detecting pure epistasis because of its high false-negative error. This poor performance can be traced back to the manner in which SAA exploits an omnibus permutation test. As stated earlier, single-locus analysis does not detect any association between a SNP and the disease in this study. Hoh et al. [11] have demonstrated that genetic association can be more significantly observed when the single-locus test statistics are combined together. Nonetheless, there is an additional requirement that each causative SNP must exhibit a marginal single-locus effect. In the current study, the association signal from each causative SNP is lower than the required threshold, leading to similar test statistics and global $p$-values for both combinations of multiple SNPs which include causative SNPs and those which exclude causative SNPs.

Both 2LOmb and exhaustive two-locus analysis technique are capable of identifying all causative SNPs. However, the size of output SNP set from $2 \mathrm{LOmb}$ is significantly smaller than that from the exhaustive two-locus analysis. Appended SNPs to the causative SNPs in the output from $2 \mathrm{LOmb}$ and those from the exhaustive two-locus analysis are erroneous SNPs. These erroneous SNPs are parts of false two-SNP units with Bonferroni-corrected $\chi^{2}$ s $p$-val- 

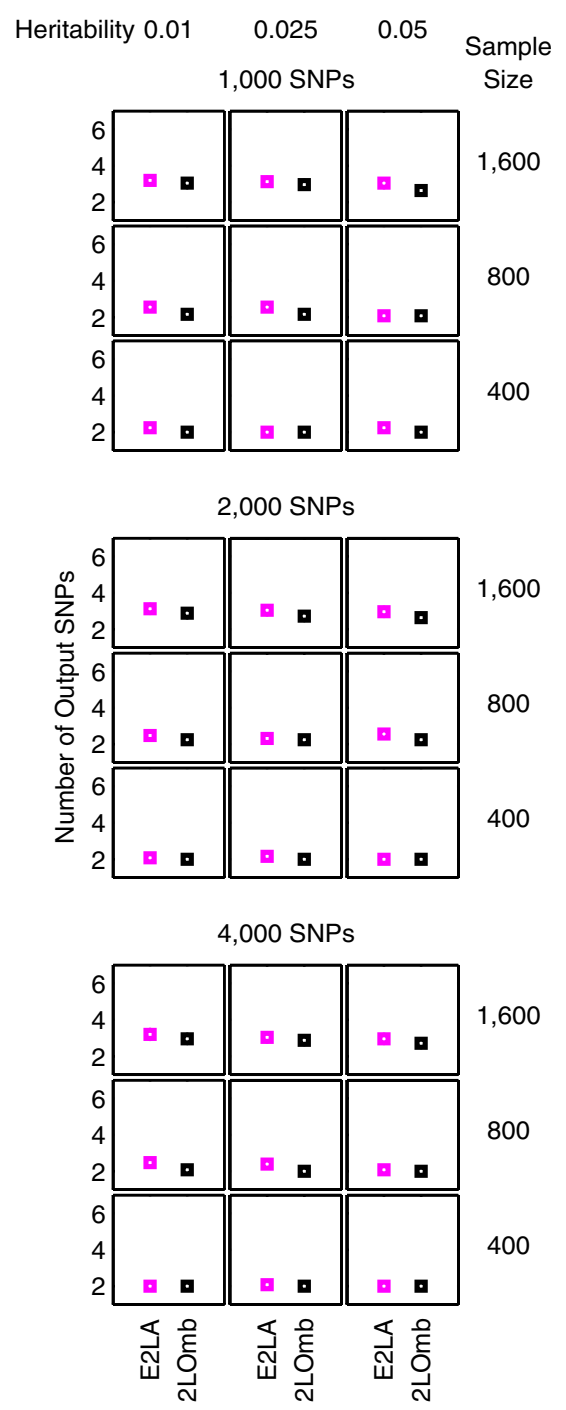

\section{Figure 3}

Performance of the exhaustive two-locus analysis and 2 LOmb in the two-locus interaction problem. The results are averaged over 25 independent simulations. Detection is declared for the exhaustive two-locus analysis and $2 \mathrm{LOmb}$ if the $p$-values used as detection indicators in their results are less than 0.05 . The results from the exhaustive two-locus analysis (E2LA) and 2LOmb are displayed using magenta and black markers, respectively. In each chart, the horizontal axis represents the detection algorithm while the vertical axis represents the number of output SNPs reported by the algorithm. All causative SNPs are present in outputs from both the exhaustive two-locus analysis and $2 \mathrm{LOmb}$ in all simulations. ues less than 0.05. A similar trend of results regarding the size of output SNP set is also observed in the benchmark trial involving the application of $2 \mathrm{LOmb}$ and exhaustive two-locus analysis to the null data. This signifies that the permutation test and the progressive search embedded in $2 \mathrm{LOmb}$ can help reducing the number of erroneous SNPs in the output.

As mentioned earlier, $2 \mathrm{LOmb}$ produces the best results among five techniques in the benchmark trial. $2 \mathrm{LOmb}$ has a low false-positive error in the null data problem and is capable of detecting all causative SNPs in every simulated data set in all three interaction problems. This performance is further strengthened by highly significant global $p$-values in $2 \mathrm{LOmb}$ results from all three interaction problems $(p<0.0001)$ and the presence of a SNP in common among some or all pairs of two-SNP units in the three- and four-locus interaction problems. Nonetheless, some of the $2 \mathrm{LOmb}$ outputs contain a few erroneous SNPs which are irrelevant to the correct association explanation. Since all three interaction problems involving different numbers of causative SNPs are investigated by varying the total number of SNPs, the sample size and the level of heritability, these parameters may influence the number of erroneous SNPs in the 2LOmb results. Similarly, the total number of SNPs and the sample size may affect the number of erroneous SNPs in the 2LOmb results from the null data problem. ANOVA reveals that the only source of variation that significantly affects the number of erroneous SNPs in the null data, two-locus interaction and three-locus interaction problems is the sample size ( $p$ $<0.000001)$. In addition, the sample size must be greater than 800 for an increase in the number of erroneous SNPs to be significant. In contrast, ANOVA reveals that two sources of variation that affect the number of erroneous SNPs in the four-locus interaction problem are the sample size $(p<0.000001)$ and the total number of SNPs $(p<$ $0.00005)$. Similar to the null data, two-locus interaction and three-locus interaction problems, the sample size in the four-locus interaction problem must be greater than 800 to create a significant increase in the number of erroneous SNPs. On the other hand, the number of erroneous SNPs appears to decrease when the total number of SNPs increases. These two sources of variation also interact with each other $(p<0.005)$. However, the interaction is most evident only when the sample size is large, i.e. when the sample size is 1,600 .

ANOVA shows that the number of erroneous SNPs in the $2 \mathrm{LOmb}$ results is influenced by the sample size and the total number of SNPs but not by the heritability. It is observed that the number of erroneous SNPs increases when the sample size is large. This counterintuitive phenomenon can be explained as follows. As $2 \mathrm{LOmb}$ com- 

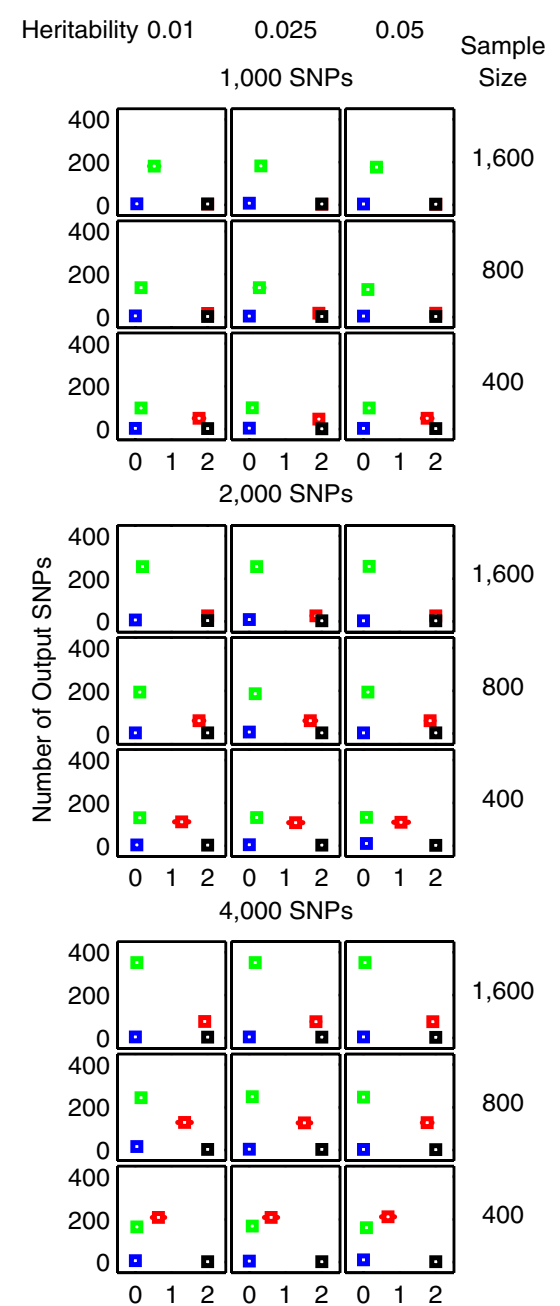

Number of Correctly-Identified Causative SNPs

\section{Figure 4}

Performance of SAA, CFS, TuRF and 2LOmb in the two-locus interaction problem. The results are averaged over 25 independent simulations. Detection is declared for SAA and 2 LOmb if the $p$-values used as detection indicators in their results are less than 0.05 . The results from SAA, CFS, TuRF and 2LOmb are displayed using blue, green, red and black markers, respectively. In each chart, the horizontal axis represents the number of correctly-identified causative SNPs while the vertical axis represents the number of output SNPs reported by the algorithm. The charts on which the red markers are invisible denote the situations in which the performance of TuRF and 2LOmb is similar. The charts in this figure are displayed using a coarser scale than the charts in Figure 3. bines $p$-values that are determined from $\chi^{2}$ tests, the number of entries for the contingency table construction is large when the sample size is large. This subsequently leads to a significantly large $\chi^{2}$ statistic and hence an extremely small $p$-value if the SNPs under consideration are causative SNPs. At the same time, the possibility that a reasonably large $\chi^{2}$ statistic and a small $p$-value can be obtained by chance from a two-SNP unit which is irrelevant to the correct association explanation also inevitably increases. With the increase in the possibility of erroneous SNP inclusion, the size of output SNP set gets bigger when the sample size is large. Another observation that appears to be counterintuitive is the reduction in the number of erroneous SNPs when the total number of SNPs increases. This phenomenon is the result of the Bonferroni correction usage. When the total number of SNPs is doubled, the Bonferroni correction factor in 2LOmb is quadrupled. A higher correction factor leads to a more stringent criterion for SNP selection. This subsequently leads to the reduction in the number of erroneous SNPs when the total number of SNPs is large.

In contrast to the first two parameters, different levels of heritability appear to have no effect on the $2 \mathrm{LOmb}$ results because all simulated data sets have balanced case-control samples and the embedded interaction models have the same architecture. For instance, a two-locus interaction model leads to zero penetrances for genotypes $A A B B$, $A A B b, A a B B, A a b b, a a B b$ and $a a b b$. Hence, the penetrances for these six genotypes are always equal to zero regardless of the heritability. On the other hand, genotypes $A A b b$, $A a B b$ and $a a B B$ have non-zero penetrances (see Methods for details). Therefore, different heritability levels certainly lead to different penetrances for genotypes $A A b b$, $A a B b$ and $a a B B$. However, the ratios between the penetrances of these three genotypes are fixed and independent of the heritability. This model description can be generalised to cover the other multi-locus interaction models. In addition, the maximum penetrance in any two-locus or multi-locus interaction models always stays below 0.1 even though the heritability is at the highest level (see Methods for details). This means that case samples are always over-sampled from affected individuals to achieve a balanced case-control data set. Since all explored heritability levels lead to the same case over-sampling pattern, the simulated data sets of which the only primary difference being the heritability levels are indistinguishable from one another. This leads to the result similarities in interaction problems with the same number of SNPs in the data set, sample size and number of causative SNPs but different levels of heritability as shown in Figures 3, 4, $5,6,7$ and 8 . The result trend is also independent of the number of simulated data sets used in the benchmark trial. 


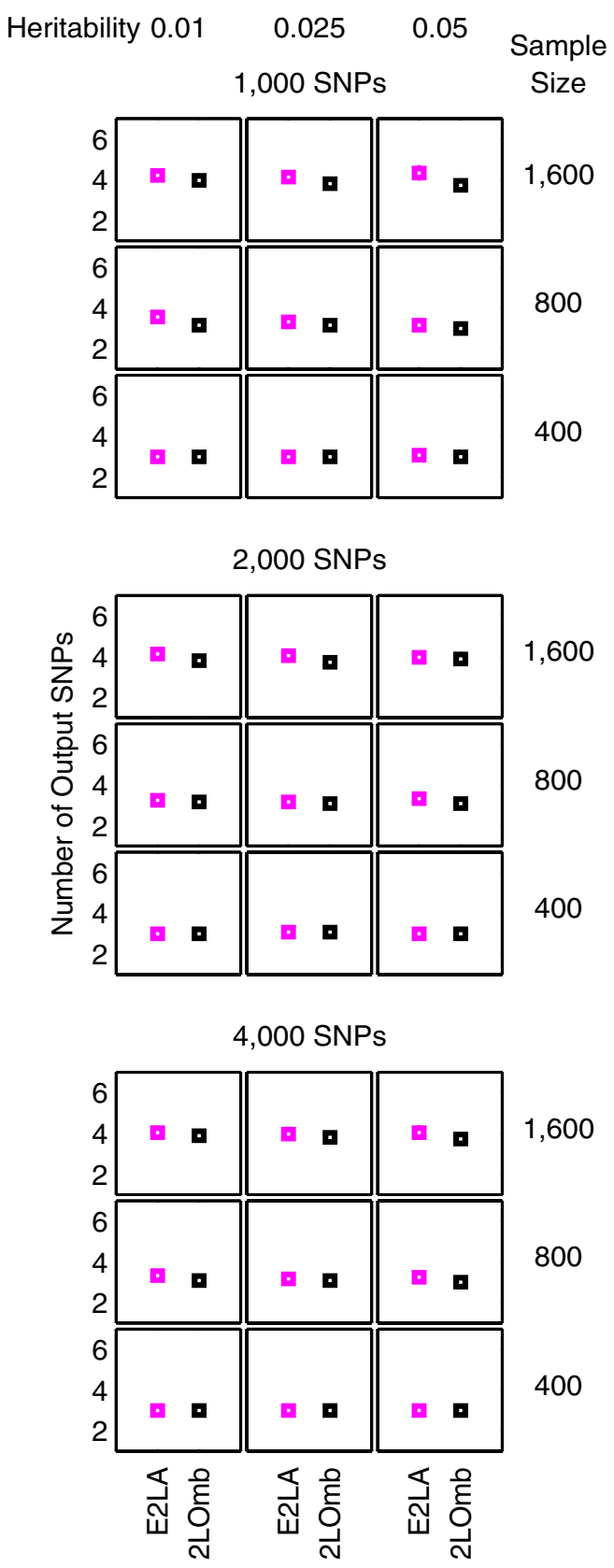

Figure 5

Performance of the exhaustive two-locus analysis and $2 \mathrm{LOmb}$ in the three-locus interaction problem. The explanation for how the results are obtained and displayed is the same as that given in Figure 3.
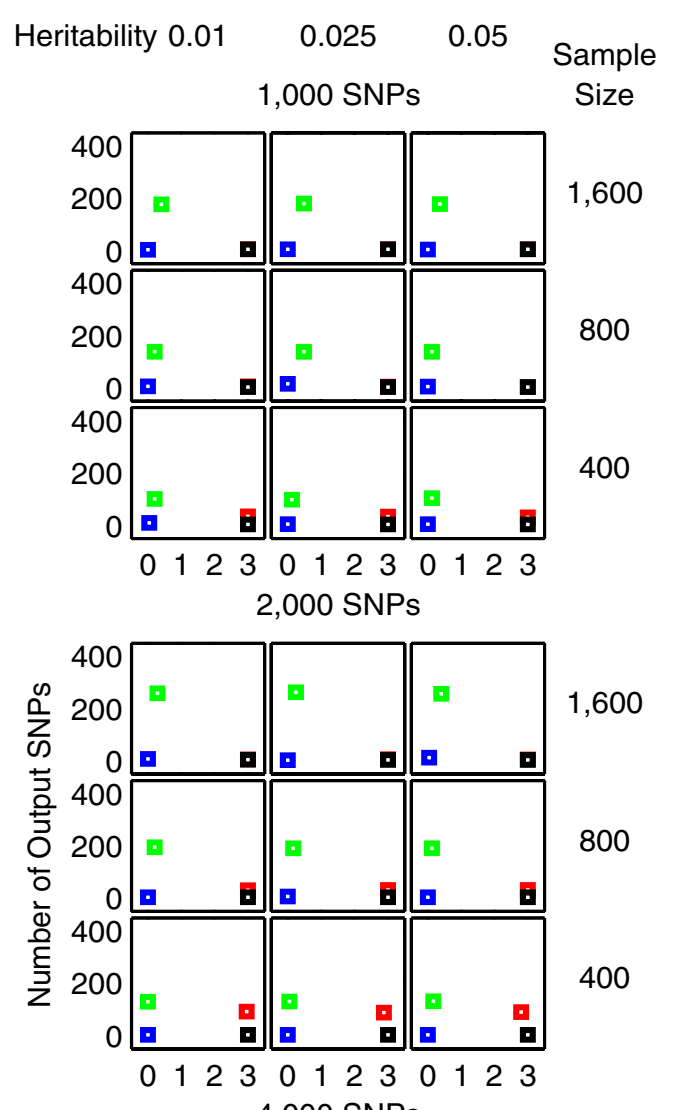

4,000 SNPs

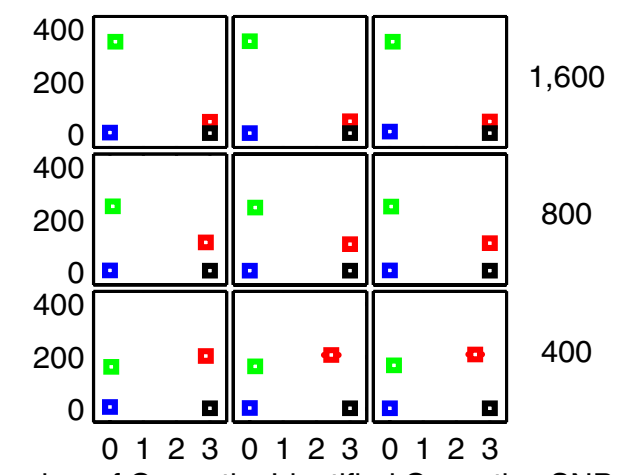

Number of Correctly-Identified Causative SNPs

\section{Figure 6}

Performance of SAA, CFS, TURF and 2LOmb in the three-locus interaction problem. The explanation for how the results are obtained and displayed is the same as that given in Figure 4. 

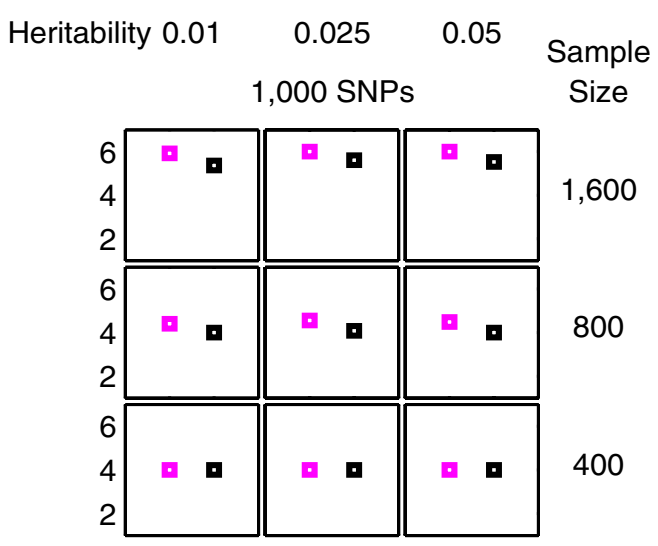

2,000 SNPs

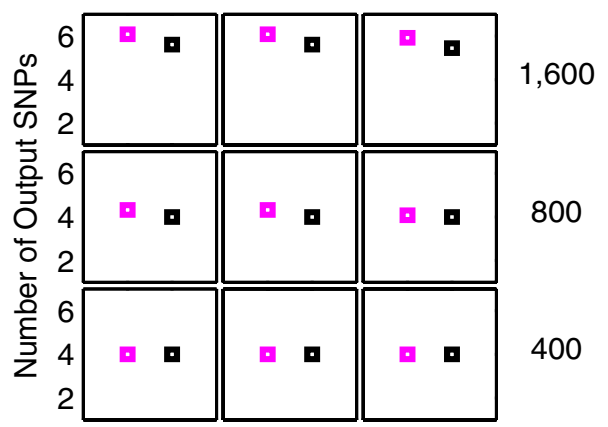

4,000 SNPs

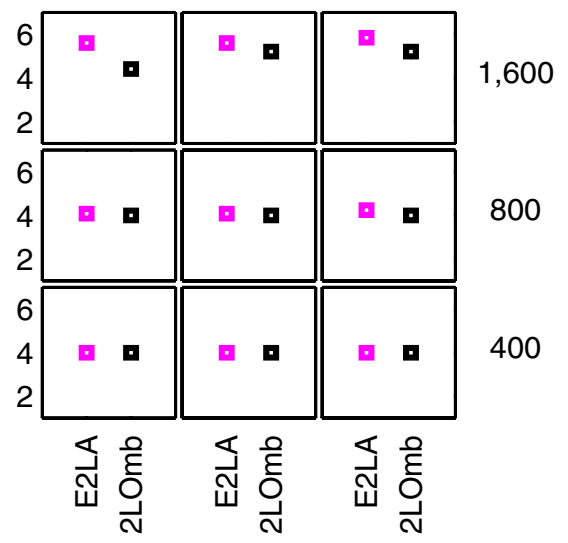

Figure 7

Performance of the exhaustive two-locus analysis and 2 LOmb in the four-locus interaction problem. The explanation for how the results are obtained and displayed is the same as that given in Figure 3.
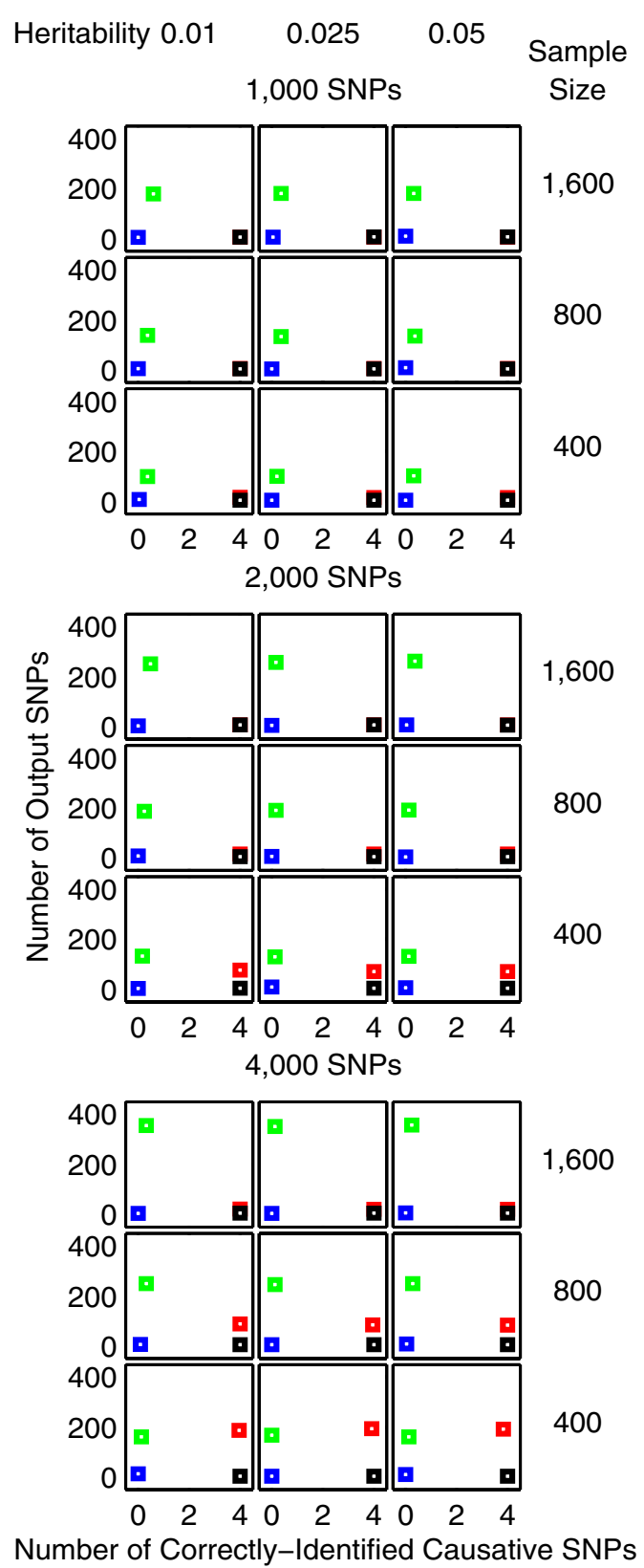

Figure 8

Performance of SAA, CFS, TuRF and 2LOmb in the four-locus interaction problem. The explanation for how the results are obtained and displayed is the same as that given in Figure 4. 
In a permutation test, the ability to differentiate between two $p$-values is influenced by the number of permutation replicates. With $t$ permutation replicates, the test declares an actual $p$-value that is less than $1 / t$ to be zero. During the progressive search for the best ensemble, the inclusion of a new two-SNP unit is accepted if this inclusion does not worsen the current result. If the number of permutation replicates is too low, the search may include erroneous two-SNP units that are irrelevant to the correct association explanation. The analysis is confirmed as the number of output SNPs from $2 \mathrm{LOmb}$ is equal to the number of causative SNPs in most of simulation results. This phenomenon suggests that the number of permutation replicates employed in this study $(t=10,000)$ is high enough to screen off most of the erroneous two-SNP units. In other words, the inclusion of these erroneous two-SNP units leads to an increase in the $p$-value by at least $1 / t$. Nonetheless, the fact that $2 \mathrm{LOmb}$ results are not entirely free from erroneous SNPs suggests that there are erroneous two-SNP units with extremely small $p$-values. It is advisable to perform a genotype relative risk calculation for the elimination of erroneous SNPs. If the presence of an erroneous two-SNP unit is suspected, its result on two-locus genotype relative risk would not be as significant as that from the other two-SNP units in the ensemble. Alternatively, an additional means for further SNP screening by other techniques such as MDR is also recommended. The chance of erroneous SNP discovery would be further minimised by employing two consecutive attribute selection techniques. The same concept has been adopted for the implementation of MDR software, in which many additional filters including a $\chi^{2}$ test, an odds ratio test, ReliefF and TuRF are available for SNP screening prior to the MDR analysis.

The two-, three- and four-locus interaction data sets which have been screened for causative SNPs by 2LOmb are subsequently subjected to MDR analysis. MDR has successfully identified all erroneous SNPs and the correct interaction models have been constructed from all data sets. The prediction accuracy from the MDR analysis is illustrated in Figure 9. It is noted that the prediction accuracy from all data sets is quite high due to the manner in which the pure epistasis model is defined [42]. Using the penetrance table for a two-locus interaction model with the heritability $=0.01$ (see Methods for details), the twolocus genotype distribution of causative SNPs in a balanced case-control sample set from simulated data with 800 samples can be estimated and shown in Figure 10.

Six genotypes in Figure 10 namely $A A B B, A A B b, A a B B$, $A a b b, a a B b$ and $a a b b$ are protective genotypes. In other words, a sample with one of these six genotypes is a control sample because the penetrances for these genotypes are zero. It is also noted that the control samples with all nine genotypes precisely follow the distribution as jointly described by independent single-locus genotype distribution from loci $\mathrm{A}$ and $\mathrm{B}$. In contrast, three remaining genotypes in Figure 10 namely $A A b b, A a B b$ and $a a B B$ are labelled as disease-predisposing genotypes because the majority of samples with these three genotypes are case samples. Samples with these genotypes may be either case or control samples because the penetrances for these genotypes are between zero and one. In fact, the probabilities that persons with these genotypes to have the disease are quite low since the penetrances for these genotypes are small. However, case samples must be over-sampled from affected individuals to ensure a balanced case-control data set because the disease prevalence for this two-locus interaction model is only 0.004975. In addition, each case sample must contain one of these three genotypes because the penetrances for the other genotypes are zero. As a result, the case samples with these genotypes do not follow the same two-locus genotype distribution as in the control samples. With six genotypes being exclusively specific to control samples and the majority of three remaining genotypes being found in case samples, the MDR prediction accuracy for the two-locus interaction model is high. This explanation can also be generalised to cover the MDR results from the other multi-locus interaction data sets.

Another advantage of using 2LOmb for SNP screening prior to the MDR analysis is the reduction in computational time for interaction detection. The computational time for $2 \mathrm{LOmb}$ to finish screening the SNPs is provided to demonstrate this strength of 2LOmb. Moreover, the computational time required to identify causative SNPs by the MDR analysis and that by the combined approach which involves SNP screening by $2 \mathrm{LOmb}$ and follows by the MDR analysis is given. The previously-described simulated data sets with causative SNPs are used to produce the computational time results from the SNP screening by $2 \mathrm{LOmb}$ and the combined approach. All possible interaction models that can be constructed from the 2LOmb outputs are explored by MDR in the combined approach. On the other hand, the data sets for the direct MDR analysis are prepared by restricting the number of SNPs in each data set to 100 . Only SNPs that are irrelevant to the correct association explanation are removed from the original simulated data sets. Furthermore, MDR only explores the interaction models that do not cover more than four SNPs in the data for this latter simulation setting. The summary of computational time required for the SNP screening by $2 \mathrm{LOmb}$ and that for both direct MDR and combined approaches to correctly identify all causative SNPs is given in Table 1 . The maximum time required by $2 \mathrm{LOmb}$ to screen SNPs in the largest data set is 419 seconds or approximately seven minutes. Moreover, the combined 2LOmb and MDR approach discovers the correct causative SNPs much faster than MDR. This time reduction is 
achieved even though the problems have been simplified for the direct MDR analysis. A direct application of MDR to the original simulated data sets is certainly impractical.

The simulated multi-locus interaction problems in this article are based on the pure epistasis model by Culverhouse et al. [42]. It is possible to capture a number of multi-locus interactions with marginal two-locus effects via a combination of two-locus analyses. However, there are many multi-locus interaction scenarios without marginal two-locus effects. In such cases, $2 \mathrm{LOmb}$ and the exhaustive two-locus analysis technique are unable to detect interactions. Among the explored techniques, TuRF and MDR have a better chance of detection. Nonetheless, TuRF functions well only when the total number of SNPs in data is small and the sample size is large enough while the total number of SNPs in data affects the practicality of direct MDR analysis.

Every attribute selection technique has a limitation in terms of the maximum numbers of samples and attributes that it can handle. Single-locus analysis techniques always have a higher limit than multi-locus analysis techniques.

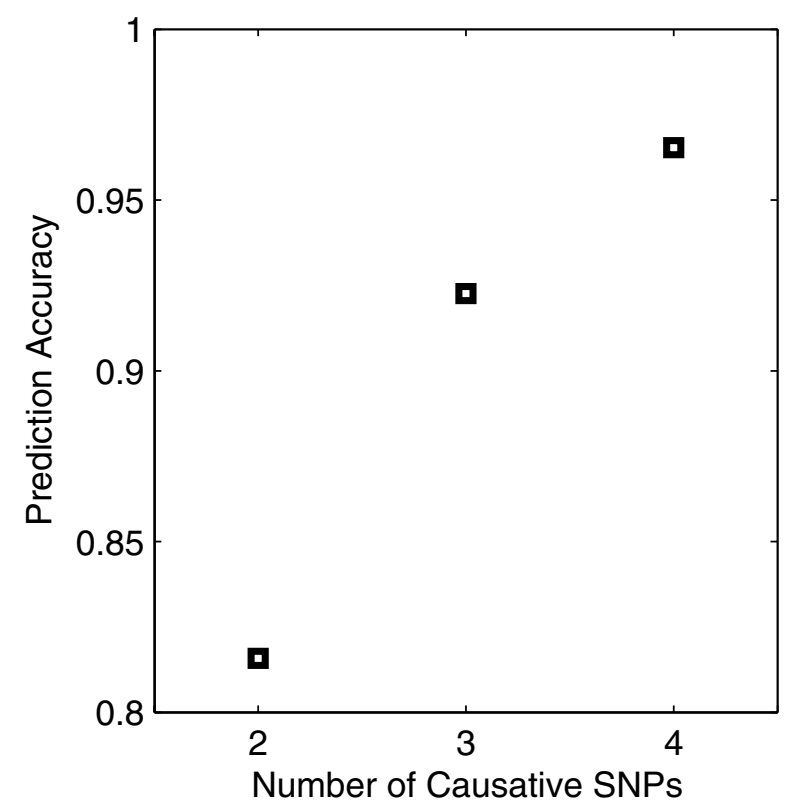

\section{Figure 9}

Prediction accuracy from the MDR analysis. A 10 -fold cross-validation strategy is applied during the accuracy evaluation. The best MDR model is located by exploring all possible SNP combinations. All erroneous SNPs, which are left over after the screening by $2 \mathrm{LOmb}$, have been successfully identified. All MDR models contain the correct number of causative SNPs. In addition, the MDR cross-validation consistency is $10 / 10$.
Because attribute subset evaluation is usually integrated into multi-locus analysis techniques, consequently the number of possible attribute subsets that can be explored is extremely large when the candidate attribute set is large. Together with a potentially large sample size, a higher computational requirement for multi-locus analysis techniques is inevitable. As a result, the direct application of multi-locus analysis techniques to a much larger data set than those presented in this article, which is usually considered in genome-wide association studies, would be impractical. However, it is reasonable to expect that both marginal single-locus and epistatic effects are present in any genome-wide data sets. A multi-stage strategy that incorporates multiple techniques, designed for different detection modes, would be more suitable to handle large data. For instance, the marginal single-locus effects should be the first priority and, as such, be detected by singlelocus analysis. Then, a special case of pure epistasis [2] or semi-purely epistatic events, in which a SNP displaying a marginal single-locus effect interacts with a SNP that exhibits no marginal single-locus effect, should be considered. Many two-locus analysis techniques have been proven to be well suited to this type of epistasis $[40,50,51]$. Finally, the detection of pure epistasis is carried out in the last stage. With the reduction of SNPs from the first stage, the chance that some multi-locus analysis techniques are applicable to the remaining SNPs increases. In addition to the multi-stage approach, a prior knowledge regarding the previously reported association can be exploited to select candidate genes based upon ontology and pathways. This practice is due to the necessity for the derivation of plausible interpretation. The screening for SNPs within or near candidate genes before the association detection also increases the chance that multi-locus analysis techniques can be applied to the remaining data.

\section{Testing with real data}

2LOmb has been applied to study a type 2 diabetes mellitus (T2D) data set, collected and investigated by the Wellcome Trust Case Control Consortium (WTCCC) [3]. The data set consists of 1,999 case samples from affected individuals in the UK and 3,004 control samples, which are the results of a merging between 1,500 samples from the UK blood services and 1,504 samples from the 1958 British birth cohort. The original genome-wide data set contains 500,568 SNPs that are obtained through the Affymetrix GeneChip $500 \mathrm{~K}$ Mapping Array Set. The SNP set is primarily reduced by screening for SNPs within and near 372 candidate genes collected by the Human Genome Epidemiology Network (HuGENet) [62]. These candidate genes cover genes from both positive and negative genetic association reports, in which studies are conducted in various ethnic groups and populations. The SNP set is further reduced by removing SNPs that exhibit 


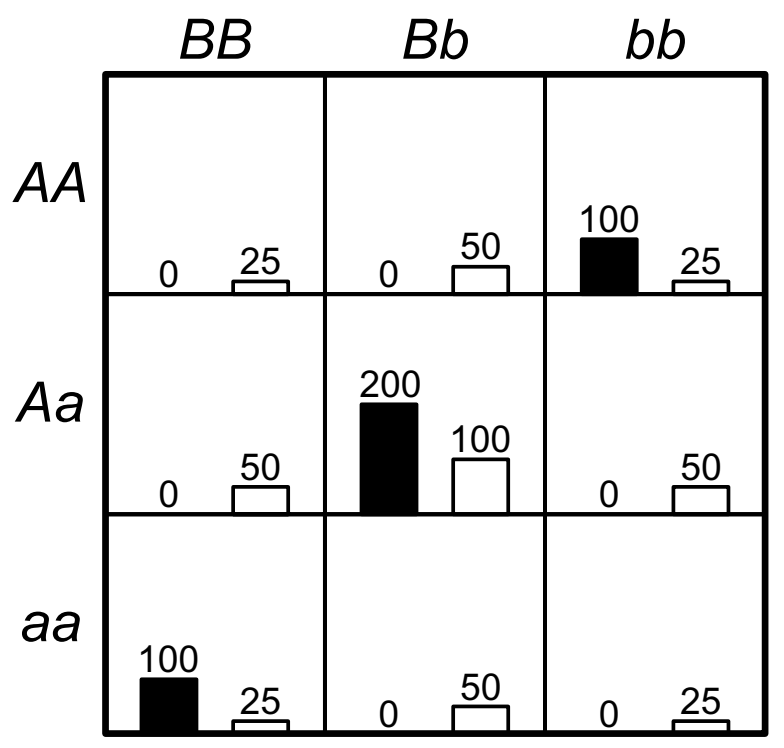

\section{Figure 10}

Genotype distribution of two causative SNPs in a balanced case-control data set with the sample size of 800. The left (black) bar in each cell represents the number of case samples while the right (white) bar represents the number of control samples. The cells with genotypes $A A B B$, $A A B b, A a B B, A a b b, a a B b$ and $a a b b$ are labelled as protective genotypes while the cells with genotypes $A A b b, A a B b$ and $a a B B$ are labelled as disease-predisposing genotypes.

strong evidence of genetic association via single-locus analysis. The final SNP set contains 7,065 SNPs from 370 candidate genes. All SNPs in the reduced data set exhibit no marginal single-locus effects (Bonferroni-corrected $\chi^{2}$ 's $p$-value $>0.05)$. Detailed description of the final SNP set is given in the supplement (see Additional file 1).

The 2LOmb search in the reduced T2D data set takes 3,456 seconds (57.6 minutes) of computational time on the Beowulf cluster. The possible genetic association is detected from 11 intronic SNPs in four genes (global $p$ value $<0.0001)$. Details of these SNPs, the two-SNP units that exhibit marginal two-locus effects and the identified genes are given in Table 2. A two-SNP unit is located in LMX1A. A two-SNP unit is also detected in PARK2. In addition, there is one SNP in common among SNPs in both GYS2 two-SNP units. Similarly, there is one common SNP among three two-SNP units located in PGM1. Nonetheless, a two-SNP unit in which each SNP is located in a different gene is absent, indicating that there is no evidence of gene-gene interactions which can be observed from the $2 \mathrm{LOmb}$ result. Linkage disequilibrium (LD) analysis is subsequently performed using a JLIN package [63] and the resulting LD patterns are illustrated in Figure 11. It is noted that there is strong LD among SNPs within each gene due to high values of $D^{\prime}$ [64] and $r^{2}$ [65]. The genotype and haplotype relative risks are then calculated and the results are presented in Tables 3, 4, 5, 6, 7, 8, 9 and 10. Haplotype inference is carried out using an expectation-maximisation method [66]. The analysis reveals that a more prominent indication of a relative risk is observed when two-SNP units are considered. It is also noted that the genotype relative risk is directly influenced by the haplotype relative risk once a genotype is phased into all possible haplotype pairs. The detection of these two-SNP units is thus believed to be the consequence of haplotype effects. An early T2D association study also reveals similar haplotype effects in FUSION data [67]. Next, an interaction dendrogram $[68,69]$ constructed from the 11 SNPs by MDR software is given in Figure 12. A strong synergistic effect between the two SNPs in PARK2 is clearly observed. In contrast, the interactions between PGM1, LMX1A, PARK2 and GYS2 are clearly absent.

Since many early genetic association studies of T2D and metabolic syndrome employ MDR analysis [43-45,47], additional MDR analysis would be useful for the comparison. The screened T2D case-control data set which contains 11 SNPs identified by $2 \mathrm{LOmb}$ is further subjected to MDR analysis. The prediction accuracy of the best MDR model is summarised in Table 11 . The model covers six SNPs in three genes: PGM1, PARK2 and GYS2. These SNPs are also present in three two-SNP units identified by $2 \mathrm{LOmb}$. It is noted that the prediction accuracy in this real data set is much less than that from the simulated data sets. Nevertheless, the attainment of low prediction accuracy does not necessarily suggest that there is no genetic association. Early works involving genetic association studies of T2D and metabolic syndrome in various populations via MDR analysis produce similar values of prediction accuracy as summarised in Table 12. The prediction accuracy by MDR from most studies is in the range of 0.5 0.6. The only genetic association study of $\mathrm{T} 2 \mathrm{D}$ that the prediction accuracy is distinctively high is conducted in a Korean population [43]. Differences in genetic background, candidate genes and selected SNPs are the main causes of variation in the genetic association results. Although MDR does not select five SNPs from the 2LOmb output, these SNPs should not be regarded as erroneous SNPs because there is strong linkage disequilibrium among SNPs in each gene. Moreover, early genotype and haplotype relative risk analysis clearly indicates that each gene, identified by $2 \mathrm{LOmb}$, plays a role in the T2D association explanation. Overall, the analysis with the methods above only confirms the positive association for PGM1, LMX1A, PARK2 and GYS2 while gene-gene interactions are clearly absent. This signifies that, for the current study, there is no interaction between each pair of the identified genes that can be described by purely epistatic two-locus interaction models. In addition, there are no interactions 
Table I: Computational time required by 2 LOmb, a combined 2 LOmb and MDR approach, and direct MDR analysis to detect interactions in simulated data sets with different sizes and different numbers of causative SNPs.

\begin{tabular}{|c|c|c|c|c|c|c|c|c|}
\hline \multirow[b]{3}{*}{$\begin{array}{c}\text { Number of } \\
\text { Causative SNPs }\end{array}$} & \multirow[b]{3}{*}{ Sample Size } & \multicolumn{7}{|c|}{ Computational Time Required by Each Approach (sec) } \\
\hline & & \multicolumn{3}{|c|}{ 2LOmb } & \multicolumn{3}{|c|}{ 2LOmb+MDR } & \multirow{2}{*}{$\begin{array}{c}\text { MDR } \\
100 \text { SNPs }\end{array}$} \\
\hline & & 1,000 SNPs & 2,000 SNPs & 4,000 SNPs & 1,000 SNPs & 2,000 SNPs & 4,000 SNPs & \\
\hline \multirow[t]{3}{*}{2} & 400 & 15 & 37 & 135 & 17 & 39 & 137 & 7,656 \\
\hline & 800 & 21 & 59 & 224 & 23 & 61 & 226 & 15,990 \\
\hline & 1,600 & 36 & 106 & 400 & 38 & 108 & 402 & 31,222 \\
\hline \multirow[t]{3}{*}{3} & 400 & 22 & 43 & 140 & 24 & 45 & 142 & 7,721 \\
\hline & 800 & 30 & 65 & 229 & 32 & 67 & 231 & 16,206 \\
\hline & 1,600 & 50 & 115 & 406 & 52 & 117 & 408 & 31,232 \\
\hline \multirow[t]{3}{*}{4} & 400 & 32 & 55 & 150 & 34 & 57 & 152 & 7,841 \\
\hline & 800 & 46 & 80 & 236 & 48 & 82 & 238 & 16,285 \\
\hline & 1,600 & 70 & 133 & 419 & 72 & 135 & 421 & 31,637 \\
\hline
\end{tabular}

Only one computing processor in a Beowulf cluster is occupied during the analysis of one data set. The test problems for the direct MDR analysis have been simplified by reducing the number of SNPs in each data set to achieve attainable computational time. The displayed time is collected from the processing of multiple independent data sets for each simulation setting. The computational time from the benchmark trial involving $2 \mathrm{LOmb}$, and the combined 2 LOmb and MDR approach is the maximum time needed by each method to detect interactions in one data set. In contrast, the computational time from the direct MDR analysis is the minimum time for the completion of interaction detection in one data set. The computational time required by $2 \mathrm{LOmb}$ for the null data problem is similar to that for the two-locus interaction problem.

between these four genes that can be described by purely epistatic multi-locus interaction models with marginal two-locus effects.

The four genes selected by $2 \mathrm{LOmb}$ regulate many pathways that involve in the disease development [70-72]. The genetic association studies involving these genes have been previously conducted in different populations. For instance, $L M X 1 A$ has been chosen as a positional and biological candidate gene for a case-control study of T2D in Pima Indians [73]. This gene is chosen as a candidate because a linkage of T2D to chromosome 1q21-q23 has been previously reported [74]. In addition, $L M X 1 A$ is one of LIM homeobox genes that are expressed in pancreas and has been shown to activate insulin gene transcription. Although SNPs have been carefully selected from the entire gene, no association between these SNPs in LMX1A and T2D has been found in this ethnic group.

PARK2 is another candidate gene that is also selected for case-control studies, based on evidence from genomewide linkage analysis [75]. A linkage of T2D in an African American population to chromosome 6q24-q27 has been previously identified [76]. Although PARK2 mainly involves in the development of Parkinson's disease, single-locus analysis reveals strong evidence of association between SNPs, which are in the vicinity of SNPs identified by $2 \mathrm{LOmb}$, and T2D in African Americans.

In contrast to $L M X 1 A$ and $P A R K 2$, which are candidate genes in typical T2D case-control studies, GYS2 is considered in a study to identify genes responsible for troglitazone-associated hepatotoxicity in Japaneses with T2D [77]. In other words, both case and control samples in the study are drawn from troglitazone-treated T2D patients, in which case patients exhibit an abnormal increase in alanine transaminase (ALT) and aspartate transaminase (AST) levels. GYS2 regulates starch and sucrose metabolism and an insulin signalling pathway. The selected SNPs in GYS2 are not found to associate with troglitazoneinduced hepatotoxicity.

Similar to the study of GYS2, the association study involving PGM1 is not carried out as a typical T2D case-control study. In fact, an attempt to identify association between PGM1 polymorphisms and obesity has been conducted among T2D affected individuals in Italy [78]. PGM1 regulates glycolysis and gluconeogenesis, starch and sucrose metabolism, galactose metabolism, a pentose phosphate pathway, and streptomycin biosynthesis. Isozyme polymorphisms $[79,80]$, which are defined through structural differences in PGM1 protein, are used instead of SNPs in the study where positive association is identified.

In summary, positive association has been reported from previous studies involving PARK2 in African Americans and PGM1 in Italians. In contrast, negative association has been reported from previous studies about $L M X 1 A$ in Pima Indians and GYS2 in Japaneses. Both GYS2 and PGM1 regulate starch and sucrose metabolism while LMX1A and PARK2 govern insulin gene transcription and Parkinson's disease development, respectively. The above discussion strengthens the importance of conducting large-scale association studies due to two main reasons. Firstly, a gene that does not contribute to the aetiology of a complex disease in one population may be important for association explanation in another population. Secondly, the absence of interacting candidate genes from a study may lead to negative association due to a lack of necessary genetic information. A two-locus interaction 
can occur between SNPs from genes that regulate one specific pathway [44] or between SNPs from genes that regulate different pathways [45]. Furthermore, a multi-locus interaction may involve both SNPs from genes that regulate the same pathway and SNPs from genes that govern different pathways. Hence, candidate genes should be selected by considering all pathways that directly and indirectly contribute to the disease development.

This study produces evidence of association between 11 intronic SNPs in PGM1, LMX1A, PARK2 and GYS2, and T2D in a UK population. Although there are other independent genome-wide T2D data sets, the association detection within these data using a similar methodology to the presented method has never been attempted because the methodology employed in the majority of genome-wide association studies is based on single-locus analysis $[3,81]$. It is recalled that each SNP explored in the reduced T2D data set exhibits no marginal single-locus effect. Hence, the most logical approach to confirm the possibility of replicating association results from the current study is to perform the same detection method on these independent data sets. This is certainly important to gain further understanding of the genetic role in T2D susceptibility.

\section{Implementation}

2LOmb is implemented in a C programming language. All functions within the program are written by the first author except the $\chi^{2}$ distribution function, which is taken from the Numerical Recipes in C [82]. The program can be compiled by Microsoft Visual Studio and GNU C compilers. The program has been successfully tested for the execution under Windows and Linux operating systems. The time required by $2 \mathrm{LOmb}$ to complete a problem containing $n$ attributes is $T(n)=\left(\begin{array}{l}n \\ 2\end{array}\right)=n ! /((n-2) ! 2 !)=n(n-1) /$ 2. 2LOmb thus has the order of $n^{2}$ time complexity (T ( $n$ ) $\left.\in O\left(n^{2}\right)\right)$. Consequently, 2LOmb can tackle problems in quadratic time. $2 \mathrm{LOmb}$ in its present form occupies one processor during the program execution. A parallel version of $2 \mathrm{LOmb}$ for genome-wide data is under development. All results included in the study are collected from the execution of computer programs in a Beowulf cluster. The computational platform consists of 12 nodes. Each node is equipped with dual Xeon $2.8 \mathrm{GHz}$ processors and $4 \mathrm{~GB}$ of main memory. The Rocks Cluster Distribution is installed on all nodes.

\section{Conclusion}

In this article, a method for detecting epistatic multi-locus interactions in case-control data is presented. The study focuses on pure epistasis [2], which cannot be detected via single-locus analysis [42]. To overcome this difficulty, the proposed method performs an omnibus permutation test [54] on ensembles of two-locus analyses and is thus referred to as $2 \mathrm{LOmb}$. The detection performance of $2 \mathrm{LOmb}$ is evaluated using both simulated and real data. From the simulation, 2LOmb produces a low false-positive error when the tests on null data of no association are performed. Furthermore, 2LOmb can identify all causative SNPs and outperforms a simple exhaustive two-locus analysis technique, a set association approach (SAA) [11], a correlation-based feature selection (CFS) technique [14] and a tuned ReliefF (TuRF) technique [16] in various interaction scenarios with marginal two-locus effects. These scenarios are set up by varying the number of causative SNPs, the number of SNPs in data, the sample size and the heritability. ANOVA reveals that the number of SNPs in data and the sample size influence the number of erroneous SNPs appended to the correctly-identified causative SNPs in the $2 \mathrm{LOmb}$ output. In contrast, the results from $2 \mathrm{LOmb}$ appear to be insensitive to the variation in heritability. After subjecting the data sets containing only SNPs that are screened by $2 \mathrm{LOmb}$ to multifactor dimensionality reduction (MDR) analysis [19], all erroneous SNPs are successfully removed. In addition, an insight into the MDR models is provided. 2LOmb is subsequently applied to a real case-control type 2 diabetes mellitus (T2D) data set, which is collected from a UK population by the Wellcome Trust Case Control Consortium (WTCCC) [3]. The original genome-wide data set is first reduced by selecting only SNPs that locate within or near 372 candidate genes reported by the Human Genome Epidemiology Network (HuGENet) [62]. In addition, the selected SNPs must exhibit no marginal single-locus effects. The final data set, which consists of 1,999 case samples and 3,004 control samples, contains 7,065 SNPs from 370 candidate genes. $2 \mathrm{LOmb}$ identifies 11 intronic SNPs that are associated with the disease. These SNPs are located in PGM1, LMX1A, PARK2 and GYS2. The 2LOmb result suggests that there is no interaction between each pair of the identified genes that can be described by purely epistatic two-locus interaction models. Moreover, there are no interactions between these four genes that can be described by purely epistatic multi-locus interaction models with marginal two-locus effects. This evidence of genetic association for these four genes leads to an alternative explanation for the aetiology of T2D in the UK population. It also implies that SNPs from genome-wide data which are usually discarded after single-locus analysis confirms the null hypothesis of no association can still be useful for genetic association studies of complex diseases.

\section{Methods Pure epistasis model}

The pure epistasis model of interest is proposed by Culverhouse et al. [42]. The model describes a restriction or constraint for penetrance of each genotype constituting the 
$D^{\prime}$
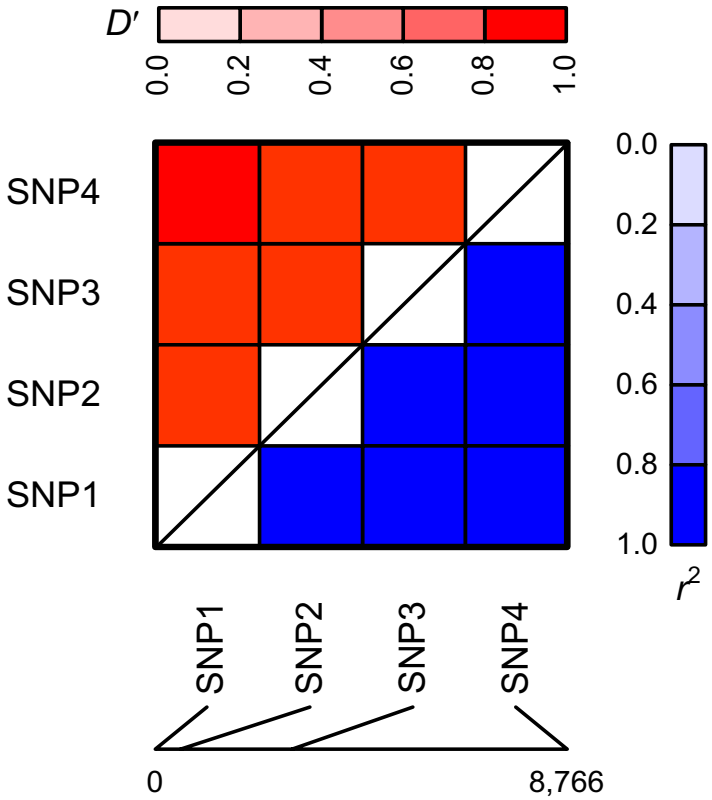

PGM1

$D^{\prime}$
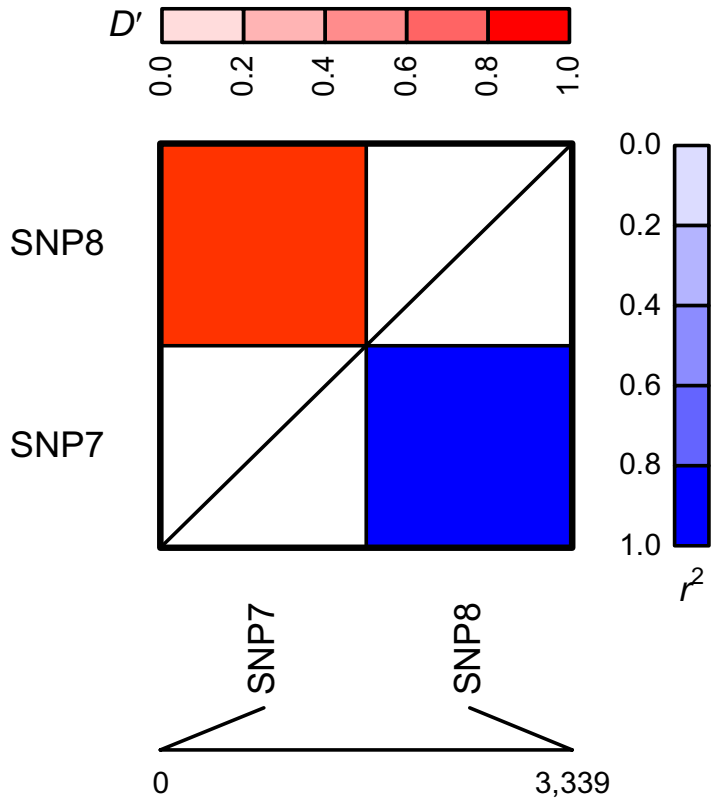

PARK2
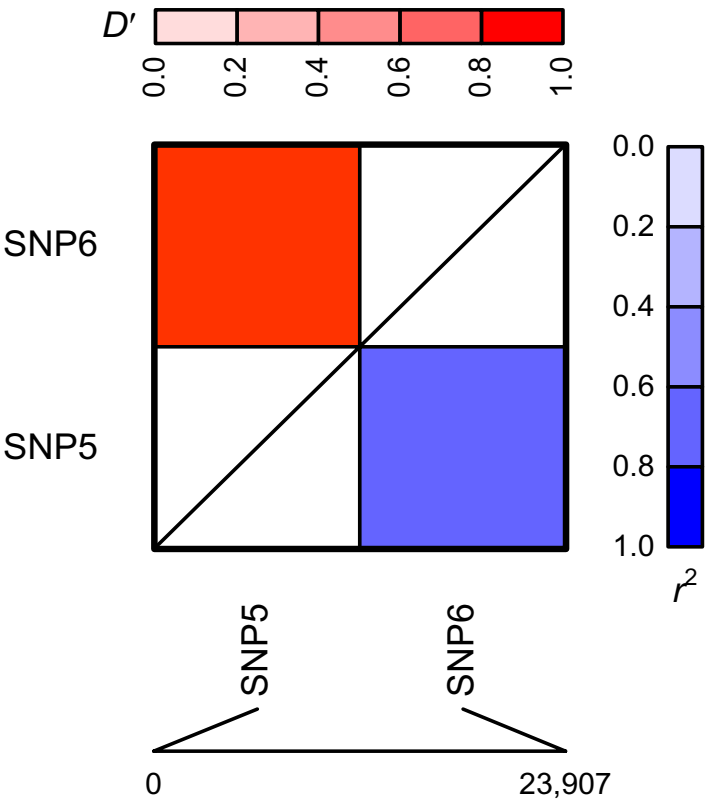

LMX1A

$D^{\prime}$

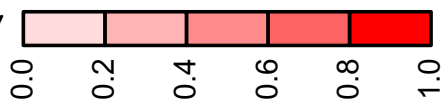

SNP11
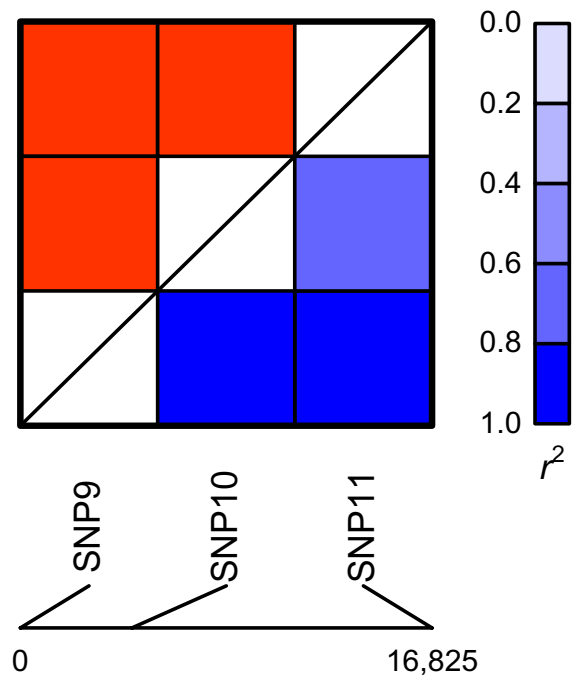

GYS2

\section{Figure I I}

Linkage disequilibrium (LD) patterns of SNPs in PGMI, LMXIA, PARK2 and GYS2. LD is explained via D' displayed in the upper triangle and $r^{2}$ displayed in the lower triangle. Dark colours indicate high values while pale colours indicate low values. Distances between SNPs are given in terms of the number of base pairs. SNPI = rs226924I, SNP2 = rs2269239, SNP3 = rs3790857, SNP4 = rs2269238, SNP5 = rs2348250, SNP6 = rs6702087, SNP7 = rs 1893551 , SNP8 = rs6924502, SNP9 $=$ rs6487236, SNPIO = rs I87II 42 and SNPII = rs 10770836. 


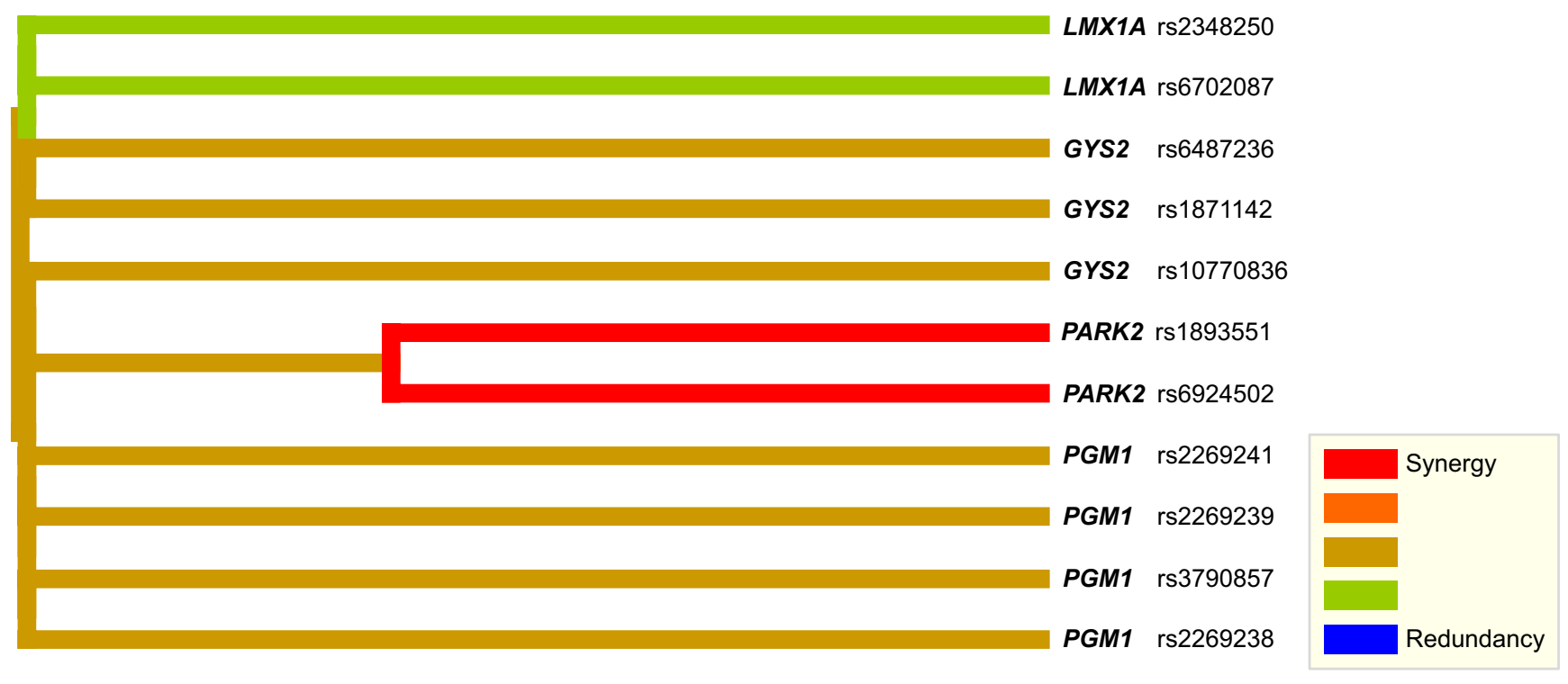

Figure 12

Interaction dendrogram produced from I I SNPs that are chosen by 2LOmb. The colours in the dendrogram comprise a spectrum of colours representing a transition from synergy to redundancy. Synergy denotes the situation in which the entropy-based interaction between two SNPs provides more information than the entropy-based correlation between the pair. Redundancy refers to the situation in which the entropy-based interaction between two SNPs provides less information than the entropy-based correlation between the pair [7].

interaction model. Consider a two-locus model that captures an interaction between loci $\mathrm{A}$ and $\mathrm{B}$, let $A$ and $a$ be the major (common) and minor (rare) alleles at locus A. Similarly, let $B$ and $b$ be the major and minor alleles at locus B. At each locus, the genotype is represented by characters 0,1 or 2 where 0 denotes a homozygous wild-type genotype ( $A A$ and $B B), 1$ denotes a heterozygous genotype $(A a$ and $B b)$ and 2 denotes a homozygous variant or homozygous mutant genotype $(a a$ and $b b) . f_{i j} \in[0,1]$ is defined as the disease penetrance of the two-locus genotype $i j$ that consists of genotype $i$ at locus A and genotype $j$ at locus $B$. The marginal penetrances $M_{A i}$ for genotype $i$ at locus $A$ and $M_{B j}$ for genotype $j$ at locus $B$ are given by

$$
M_{A i}=p_{B}^{2} f_{i 0}+2 p_{B}\left(1-p_{B}\right) f_{i 1}+\left(1-p_{B}\right)^{2} f_{i 2}, i \in\{0,1,2\}
$$

and

$$
M_{B j}=p_{A}^{2} f_{0 j}+2 p_{A}\left(1-p_{A}\right) f_{1 j}+\left(1-p_{A}\right)^{2} f_{2 j}, j \in\{0,1,2\} ，
$$

where $p_{A}$ and $p_{B}$ are the major allele frequencies. Equations 4 and 5 are usually represented by a penetrance table as illustrated in Table 13. The two-locus interaction model is a pure epistasis model if

\begin{tabular}{|c|c|c|}
\hline Gene & Chromosome and Location & Two-SNP Unit in the Ensemble \\
\hline \multirow[t]{3}{*}{ PGMI (phosphoglucomutase I) } & $|p 3|$ & (rs226924l, rs3790857) \\
\hline & & (rs2269239, rs3790857) \\
\hline & & (rs3790857, rs2269238) \\
\hline LMXIA (LIM homeobox transcription factor I, alpha) & $1 \mathrm{q} 22-\mathrm{q} 23$ & (rs2348250, rs6702087) \\
\hline PARK2 (Parkinson disease (autosomal recessive, juvenile) 2, parkin) & $6 q 25.2-q 27$ & (rs189355I, rs6924502) \\
\hline \multirow[t]{2}{*}{ GYS2 (glycogen synthase 2 (liver)) } & $|2 p| 2.2$ & (rs6487236, rs $187 \mid 142)$ \\
\hline & & (rs|87|I42, rs 10770836$)$ \\
\hline
\end{tabular}

Table 2: 2LOmb identifies II intronic SNPs, which are located in four genes, from the reduced T2D data.

Association between these SNPs and the disease is possible (global p-value $<0.000 \mathrm{I}$ ). Seven two-SNP units are present in the ensemble where each unit contains a pair of SNPs from the same gene. 
Table 3: Genotype relative risk evaluated from genotype distribution of SNPs in PGMI.

\begin{tabular}{|c|c|c|c|c|c|}
\hline \multirow[b]{2}{*}{ SNP } & \multirow[b]{2}{*}{ Genotype } & \multicolumn{2}{|c|}{ Frequency } & \multirow[b]{2}{*}{ Relative Risk } & \multirow[b]{2}{*}{$95 \% \mathrm{Cl}$} \\
\hline & & Case & Control & & \\
\hline \multirow[t]{3}{*}{ SNPI } & 0 & 0.6513 & 0.6528 & 0.9977 & (0.9573-1.0399) \\
\hline & 1 & 0.3082 & 0.3076 & 1.0018 & (0.9204-I.0905) \\
\hline & 2 & 0.0405 & 0.0396 & 1.0229 & (0.7757-I.3488) \\
\hline \multirow[t]{3}{*}{ SNP2 } & 0 & 0.6493 & 0.6521 & 0.9957 & $(0.9552-1.0379)$ \\
\hline & 1 & 0.3087 & 0.3079 & 1.0024 & $(0.9209-1.0910)$ \\
\hline & 2 & 0.0420 & 0.0399 & 1.0519 & $(0.8006-1.3822)$ \\
\hline \multirow[t]{3}{*}{ SNP3 } & 0 & 0.6153 & 0.6568 & 0.9368 & $(0.8972-0.9782)$ \\
\hline & 1 & 0.3472 & 0.3056 & 1.1361 & $(1.0479-1.2316)$ \\
\hline & 2 & 0.0375 & 0.0376 & 0.9974 & $(0.7490-I .328 I)$ \\
\hline \multirow[t]{3}{*}{ SNP4 } & 0 & 0.6638 & 0.6668 & 0.9956 & $(0.9564-1.0364)$ \\
\hline & 1 & 0.2991 & 0.2969 & 1.0074 & (0.9237-1.0988) \\
\hline & 2 & 0.0370 & 0.0363 & 1.0202 & $(0.7636-1.363 I)$ \\
\hline \multirow[t]{7}{*}{ (SNPI, SNP3) } & 00 & 0.6043 & 0.6448 & 0.9372 & $(0.8966-0.9796)$ \\
\hline & 0 I & 0.0470 & 0.0080 & 5.8858 & $(3.7730-9.1816)$ \\
\hline & 10 & 0.0110 & 0.0120 & 0.9183 & $(0.5420-1.5561)$ \\
\hline & I I & 0.2961 & 0.2943 & 1.0064 & $(0.9222-1.0983)$ \\
\hline & 12 & 0.0010 & 0.0013 & 0.7514 & $(0.1378-4.0984)$ \\
\hline & 21 & 0.0040 & 0.0033 & 1.2022 & $(0.4753-3.0408)$ \\
\hline & 22 & 0.0365 & 0.0363 & 1.0064 & $(0.7523-1.3463)$ \\
\hline \multirow[t]{7}{*}{ (SNP2, SNP3) } & 00 & 0.6038 & 0.6448 & 0.9364 & $(0.8958-0.9789)$ \\
\hline & 01 & 0.0455 & 0.0073 & 6.2159 & (3.9I54-9.868I) \\
\hline & 10 & 0.0115 & 0.0117 & 0.9875 & $(0.5853-1.6661)$ \\
\hline & 11 & 0.2966 & 0.2949 & 1.0058 & $(0.9218-1.0975)$ \\
\hline & 12 & 0.0005 & 0.0013 & 0.3757 & $(0.0420-3.3588)$ \\
\hline & 21 & 0.0050 & 0.0033 & 1.5028 & $(0.6266-3.6038)$ \\
\hline & 22 & 0.0370 & 0.0363 & 1.0202 & $(0.7636-1.363 I)$ \\
\hline \multirow{6}{*}{ (SNP3, SNP4) } & 00 & 0.6138 & 0.6551 & 0.9369 & $(0.897 \mathrm{I}-0.9785)$ \\
\hline & 01 & 0.0015 & 0.0017 & 0.9017 & $(0.2157-3.7686)$ \\
\hline & 10 & 0.0500 & 0.0117 & 4.2936 & $(2.9340-6.283 I)$ \\
\hline & 11 & $0.297 \mid$ & 0.2936 & 1.0121 & $(0.9274-1.1044)$ \\
\hline & 21 & 0.0005 & 0.0017 & 0.3006 & $(0.035 \mathrm{I}-2.5706)$ \\
\hline & 22 & 0.0370 & 0.0360 & 1.0297 & $(0.7702-1.3765)$ \\
\hline
\end{tabular}

The relative risk is calculated from the ratio between the probability of a genotype of interest occurring in the case group and that of the same genotype occurring in the control group. Only the relative risks based on genotypic information from each SNP and SNP pairs identified by 2LOmb are considered. Characters $0, I$ and 2 represent different genotypes at each locus where 0 denotes a homozygous wild-type genotype, I denotes a heterozygous genotype and 2 denotes a homozygous variant or homozygous mutant genotype. The relative risk displayed in boldface is statistically significant. The major/minor alleles for rs226924I, rs2269239, rs3790857 and rs2269238 are T/C, G/C, C/T and G/T, respectively. The allelic information is extracted from the original T2D data. SNPI $=$ rs226924I, SNP2 $=$ rs2269239, SNP3 $=$ rs3790857 and SNP4 = rs2269238.

$$
M_{A i}=M_{B j}=K, \forall i, j \in\{0,1,2\},
$$

where $K$ is the disease prevalence. Obviously, many combinations of penetrance $f_{i j}$ satisfy the condition given in equation 6. Culverhouse et al. [42] suggest that a pure epistasis model with the maximum heritability is particularly useful in association studies. The heritability $\left(h^{2}\right)$ of the two-locus interaction model is defined by

$$
h^{2}=V_{I} / V_{T}
$$

where $V_{T}=K(1-K)$ is the total variance of the dichotomous phenotypes in the population and $V_{I}$ is the epistatic variation attributable to the genotypes. $V_{I}$ is defined by

$$
\begin{aligned}
V_{I}= & p_{A}^{2} p_{B}^{2}\left(f_{00}-K\right)^{2}+2 p_{A}^{2} p_{B}\left(1-p_{B}\right)\left(f_{01}-K\right)^{2}+p_{A}^{2}\left(1-p_{B}\right)^{2}\left(f_{02}-K\right)^{2} \\
& +2 p_{A}\left(1-p_{A}\right) p_{B}^{2}\left(f_{10}-K\right)^{2}+4 p_{A}\left(1-p_{A}\right) p_{B}\left(1-p_{B}\right)\left(f_{11}-K\right)^{2}+2 p_{A}\left(1-p_{A}\right)\left(1-p_{B}\right)^{2}\left(f_{12}-K\right)^{2} \\
& +\left(1-p_{A}\right)^{2} p_{B}^{2}\left(f_{20}-K\right)^{2}+2\left(1-p_{A}\right)^{2} p_{B}\left(1-p_{B}\right)\left(f_{21}-K\right)^{2}+\left(1-p_{A}\right)^{2}\left(1-p_{B}\right)^{2}\left(f_{22}-K\right)^{2} .
\end{aligned}
$$

The search for feasible penetrance $f_{i j}$ that also maximises the heritability or other variance-based objectives can be treated as a constraint optimisation problem. Many algorithms including a double description method [42] and a genetic algorithm [83] have been proven to be suitable for the task.

Culverhouse et al. [42] have identified the maximum heritability of purely epistatic two-locus and multi-locus interaction models for various values of disease prevalence. For instance, the maximum heritability of a two- 
Table 4: Haplotype relative risk evaluated from genotype distribution of SNPs in PGMI.

\begin{tabular}{|c|c|c|c|c|c|}
\hline \multirow[b]{2}{*}{ SNP } & \multirow[b]{2}{*}{ Allele and Haplotype } & \multicolumn{2}{|c|}{ Frequency } & \multirow[b]{2}{*}{ Relative Risk } & \multirow[b]{2}{*}{$95 \% \mathrm{Cl}$} \\
\hline & & Case & Control & & \\
\hline \multirow[t]{2}{*}{ SNPI } & 0 & 0.8054 & 0.8066 & 0.9985 & $(0.9791-1.0183)$ \\
\hline & I & 0.1946 & 0.1934 & 1.0061 & $(0.9274-1.0916)$ \\
\hline \multirow[t]{2}{*}{ SNP2 } & 0 & 0.8037 & 0.8061 & 0.9970 & $(0.9775-1.0168)$ \\
\hline & I & 0.1963 & 0.1939 & 1.0126 & $(0.9336-1.0982)$ \\
\hline \multirow[t]{2}{*}{ SNP3 } & 0 & 0.7889 & 0.8096 & 0.9744 & $(0.9550-0.9943)$ \\
\hline & 1 & 0.2111 & 0.1904 & 1.1087 & $(1.0240-1.2003)$ \\
\hline \multirow[t]{2}{*}{ SNP4 } & 0 & 0.8134 & 0.8152 & 0.9977 & $(0.9789-1.0170)$ \\
\hline & 1 & 0.1866 & 0.1848 & 1.0100 & $(0.9288-1.0982)$ \\
\hline \multirow[t]{4}{*}{ (SNPI, SNP3) } & 00 & 0.7812 & 0.8019 & 0.9742 & $(0.9543-0.9945)$ \\
\hline & $0 \mathrm{I}$ & 0.0242 & 0.0047 & 5.1533 & $(3.3946-7.823 I)$ \\
\hline & 10 & 0.0077 & 0.0077 & 1.0000 & $(0.6349-1.5752)$ \\
\hline & I I & 0.1869 & 0.1857 & 1.0064 & $(0.9257-I .094 I)$ \\
\hline \multirow[t]{4}{*}{ (SNP2, SNP3) } & 00 & 0.7804 & 0.8017 & 0.9734 & $(0.9535-0.9938)$ \\
\hline & 01 & 0.0232 & 0.0044 & 5.3216 & (3.4557-8.1949) \\
\hline & 10 & 0.0085 & 0.0079 & 1.0758 & $(0.6930-1.6701)$ \\
\hline & 11 & 0.1879 & 0.1861 & 1.0099 & $(0.9291-1.0977)$ \\
\hline \multirow[t]{4}{*}{ (SNP3, SNP4) } & 00 & 0.7881 & 0.8086 & 0.9747 & $(0.9552-0.9946)$ \\
\hline & 01 & 0.0008 & 0.0010 & 0.7661 & $(0.1943-3.0205)$ \\
\hline & 10 & 0.0253 & 0.0067 & 3.7936 & $(2.6367-5.4582)$ \\
\hline & II & 0.1858 & 0.1837 & 1.0113 & $(0.9298-1.0999)$ \\
\hline
\end{tabular}

The relative risk is calculated from the ratio between the probability of a haplotype of interest occurring in the case group and that of the same haplotype occurring in the control group. Haplotype inference is carried out using an expectation-maximisation method. Only the relative risks based on genotypic information from each SNP and SNP pairs identified by 2 LOmb are considered. Characters 0 and I represent different alleles at each locus where 0 and I denote major and minor alleles, respectively. The relative risk displayed in boldface is statistically significant. The allelic information for each SNP is given in Table 3. SNPI = rs226924I, SNP2 = rs2269239, SNP3 = rs3790857 and SNP4 = rs2269238.

locus interaction model for $p_{A}=p_{B}=0.5$ with the penetrances in Table 14 is

$$
h_{\max }^{2}(K)=2 K /(1-K), K \in(0,1 / 4] .
$$

When a two-locus interaction model is expanded into a multi-locus interaction model, the marginal penetrance equality constraint must be extended to cover all loci. Fur- thermore, the expression for $V_{I}$ must also be expanded to cover additional genotypes while the expression for $V_{T}$ remains unchanged. With the necessary model expansion, the maximum heritability of a three-locus interaction model for $p_{A}=p_{B}=p_{C}=0.5$ with the penetrances in Table 15 is given by

$$
h_{\max }^{2}(K)=9 K /(1-K), K \in(0,1 / 16] .
$$

\begin{tabular}{|c|c|c|c|c|c|}
\hline \multirow[b]{2}{*}{ SNP } & \multirow[b]{2}{*}{ Genotype } & \multicolumn{2}{|c|}{ Frequency } & \multirow[b]{2}{*}{ Relative Risk } & \multirow[b]{2}{*}{$95 \% \mathrm{Cl}$} \\
\hline & & Case & Control & & \\
\hline \multirow[t]{3}{*}{ SNP5 } & 0 & 0.8429 & 0.8642 & 0.9754 & $(0.9526-0.9987)$ \\
\hline & 1 & 0.1531 & 0.1315 & 1.1642 & $(1.0140-1.3366)$ \\
\hline & 2 & 0.0040 & 0.0043 & 0.9248 & $(0.3840-2.227 I)$ \\
\hline \multirow[t]{3}{*}{ SNP6 } & 0 & 0.8799 & 0.8492 & 1.0362 & $(1.0135-1.0594)$ \\
\hline & I & 0.1161 & 0.1465 & 0.7924 & $(0.6829-0.9193)$ \\
\hline & 2 & 0.0040 & 0.0043 & 0.9248 & $(0.3840-2.227 I)$ \\
\hline \multirow[t]{5}{*}{ (SNP5, SNP6) } & 00 & 0.8329 & 0.8299 & 1.0036 & $(0.9784-1.0295)$ \\
\hline & 01 & 0.0100 & 0.0343 & 0.2918 & $(0.1814-0.4695)$ \\
\hline & 10 & 0.0470 & 0.0193 & 2.4355 & $(1.7644-3.3618)$ \\
\hline & 11 & 0.1061 & 0.1119 & 0.9482 & $(0.8061-1.1153)$ \\
\hline & 22 & 0.0040 & 0.0040 & 1.0018 & $(0.4103-2.4465)$ \\
\hline
\end{tabular}

Table 5: Genotype relative risk evaluated from genotype distribution of SNPs in LMXIA.

The explanation for how the relative risks are obtained and displayed is the same as that given in Table 3. The major/minor alleles for $\mathrm{rs} 2348250$ and rs6702087 are G/A and G/C, respectively. The allelic information is extracted from the original T2D data. SNP5 = rs2348250 and SNP6 = rs6702087. 
Table 6: Haplotype relative risk evaluated from genotype distribution of SNPs in LMXIA.

\begin{tabular}{|c|c|c|c|c|c|}
\hline \multirow[b]{2}{*}{ SNP } & \multirow[b]{2}{*}{ Allele and Haplotype } & \multicolumn{2}{|c|}{ Frequency } & \multirow[b]{2}{*}{ Relative Risk } & \multirow[b]{2}{*}{$95 \% \mathrm{Cl}$} \\
\hline & & Case & Control & & \\
\hline \multirow[t]{2}{*}{ SNP5 } & 0 & 0.9195 & 0.9299 & 0.9887 & $(0.9774-1.0002)$ \\
\hline & I & 0.0805 & 0.0701 & 1.1494 & $(0.9997-1.3214)$ \\
\hline \multirow[t]{2}{*}{ SNP6 } & 0 & 0.9380 & 0.9224 & 1.0168 & (1.0059-1.0279) \\
\hline & I & 0.0620 & 0.0776 & 0.7997 & $(0.6892-0.9280)$ \\
\hline \multirow[t]{4}{*}{ (SNP5, SNP6) } & 00 & 0.9143 & 0.9124 & 1.0021 & $(0.9898-1.0145)$ \\
\hline & 01 & $0.005 I$ & 0.0175 & $0.293 I$ & $(0.1829-0.4697)$ \\
\hline & 10 & 0.0236 & 0.0100 & 2.3640 & $(1.7 \mid 50-3.2585)$ \\
\hline & II & 0.0569 & 0.0601 & 0.9472 & $(0.8064-|| I \mid 27)$. \\
\hline
\end{tabular}

The explanation for how the relative risks are obtained and displayed is the same as that given in Table 4. The allelic information for each SNP is given in Table 5. SNP5 $=$ rs2348250 and SNP6 $=$ rs6702087.

Similarly, the maximum heritability of a four-locus interaction model for $p_{A}=p_{B}=p_{C}=p_{D}=0.5$ with the penetrances in Table 16 is

$$
h_{\max }^{2}(K)=35 K /(1-K), K \in(0,1 / 64] .
$$

Additional details about the maximum heritability and the corresponding two-locus and multi-locus penetrance tables for other values of disease prevalence can be found in Culverhouse et al. [42]. In this article, the simulated data sets are generated to achieve the maximum heritability of $0.01,0.025$ and 0.05 . The values of disease prevalence that lead to the target heritability for two-, three- and four-locus interaction models are given in Table 17.

\section{genomeSIM}

genomeSIM is a simulation package for generating casecontrol samples in large-scale and genome-wide association studies [60]. The package is capable of producing many realistic scenarios, which can be observed in a population and genetic samples, including linkage disequilibrium, phenocopy and genotyping errors. The case/control status of each sample is determined from the penetrancebased genetic models or interaction models. As a result, the package can accommodate many epistasis models including the one proposed by Culverhouse et al. [42]. A data set can be produced via two modes: a populationbased simulation and a probability-based simulation. In the population-based simulation, an initial population is generated according to the predefined allele frequency of each SNP. Then further generations are created by crossing the genotype strings within successive generations until the specified number of generations is reached. The resulting data set contains a population-dependent case and control samples that follow a forward-time simulation strategy. In contrast, genotype strings are incrementally generated without any string crossing for only one generation in the probability-based simulation. The creation of new strings is terminated only when the desired numbers

Table 7: Genotype relative risk evaluated from genotype distribution of SNPs in PARK2.

\begin{tabular}{|c|c|c|c|c|c|}
\hline \multirow[b]{2}{*}{ SNP } & \multirow[b]{2}{*}{ Genotype } & \multicolumn{2}{|c|}{ Frequency } & \multirow[b]{2}{*}{ Relative Risk } & \multirow[b]{2}{*}{$95 \% \mathrm{Cl}$} \\
\hline & & Case & Control & & \\
\hline \multirow[t]{3}{*}{ SNP7 } & 0 & 0.4802 & 0.5110 & 0.9398 & $(0.8873-0.9954)$ \\
\hline & 1 & 0.4322 & 0.4041 & 1.0695 & $(1.0008-1.1429)$ \\
\hline & 2 & 0.0875 & 0.0849 & 1.0313 & $(0.858 \mathrm{I}-1.2395)$ \\
\hline \multirow[t]{3}{*}{ SNP8 } & 0 & 0.4892 & 0.4923 & 0.9937 & $(0.9380-1.0527)$ \\
\hline & I & 0.4087 & 0.4121 & 0.9917 & $(0.9267-1.0613)$ \\
\hline & 2 & 0.1021 & 0.0955 & 1.0682 & $(0.9009-1.2665)$ \\
\hline \multirow[t]{8}{*}{ (SNP7, SNP8) } & 00 & 0.4492 & 0.4844 & 0.9275 & $(0.8726-0.9858)$ \\
\hline & 01 & 0.0285 & 0.0260 & 1.0982 & $(0.784 \mid-I .5380)$ \\
\hline & 02 & 0.0025 & 0.0007 & 3.7569 & $(0.7296-19.3450)$ \\
\hline & 10 & 0.0400 & 0.0080 & 5.0092 & (3.1856-7.8767) \\
\hline & 11 & 0.3792 & 0.3848 & 0.9854 & $(0.9169-1.0590)$ \\
\hline & 12 & 0.0130 & 0.0113 & 1.1492 & $(0.6918-1.9089)$ \\
\hline & 21 & 0.0010 & 0.0013 & 0.7514 & $(0.1378-4.0984)$ \\
\hline & 22 & 0.0865 & 0.0836 & 1.0358 & $(0.8606-1.2465)$ \\
\hline
\end{tabular}

The explanation for how the relative risks are obtained and displayed is the same as that given in Table 3. The major/minor alleles for $\mathrm{rs} / 89355 \mathrm{I}$ and rs6924502 are G/A and T/C, respectively. The allelic information is extracted from the original T2D data. SNP7 = rs I89355I and SNP8 = rs6924502. 
Table 8: Haplotype relative risk evaluated from genotype distribution of SNPs in PARK2.

\begin{tabular}{lccccc}
\hline \multirow{2}{*}{ SNP } & Allele and Haplotype & Case & Control & Relative Risk & \multicolumn{2}{c}{$\mathbf{9 5 \%}$ Cl } \\
\hline SNP7 & 0 & 0.6963 & 0.7130 & 0.9766 & $(0.9515-1.0023)$ \\
& 1 & 0.3037 & 0.2870 & 1.0582 & $(0.9950-1.1254)$ \\
SNP8 & 0 & 0.6936 & 0.6984 & 0.9931 & $(0.9672-1.0198)$ \\
(SNP7, SNP8) & 1 & 0.3064 & 0.3016 & 1.0159 & $(0.9563-1.0793)$ \\
& 00 & 0.6726 & 0.6937 & 0.9696 & $(0.9434-0.9966)$ \\
& 01 & 0.0238 & 0.0194 & 1.2248 & $(0.9369-1.6011)$ \\
& 10 & $\mathbf{0 . 0 2 1 0}$ & $\mathbf{0 . 0 0 4 8}$ & $\mathbf{4 . 4 2 1 5}$ & $\mathbf{( 2 . 8 9 7 2 - 6 . 7 4 8 0 )}$ \\
& 11 & 0.2826 & 0.2822 & 1.0016 & $(0.9397-1.0675)$ \\
\hline
\end{tabular}

The explanation for how the relative risks are obtained and displayed is the same as that given in Table 4. The allelic information for each SNP is given in Table 7. SNP7 $=$ rs $189355 \mathrm{I}$ and SNP8 $=$ rs6924502.

of case and control samples are obtained. In this study, the probability-based simulation is used to produce all case and control samples where the simulation parameter setting is given in the supplement (see Additional file 2). genomeSIM is available upon request to Scott M. Dudek at the Vanderbilt University dudek@chgr.mc.vanderbilt.edu.

\section{Set association approach}

A set association approach (SAA) is an association detection technique based on an omnibus permutation test on sets of candidate SNPs [11]. The test captures information about genotyping errors, deviation from Hardy-Weinberg equilibrium (HWE) and allelic association. In the first step, the genotype distribution for each SNP in the control samples is checked for HWE. Then, the number of SNPs that is to be excluded from the study $\left(n_{d}\right)$ is set to the number of SNPs in the control samples that deviate from HWE. Two test statistics are subsequently calculated for each SNP: an allelic association statistic and a statistic for the deviation from HWE of each SNP in the case samples. The allelic association statistic is a $\chi^{2}$ statistic which is cal-

Table 9: Genotype relative risk evaluated from genotype distribution of SNPs in GYS2.

\begin{tabular}{|c|c|c|c|c|c|}
\hline \multirow[b]{2}{*}{ SNP } & \multirow[b]{2}{*}{ Genotype } & \multicolumn{2}{|c|}{ Frequency } & \multirow[b]{2}{*}{ Relative Risk } & \multirow[b]{2}{*}{$95 \% \mathrm{Cl}$} \\
\hline & & Case & Control & & \\
\hline \multirow[t]{3}{*}{ SNP9 } & 0 & 0.6473 & 0.6575 & 0.9846 & $(0.9447-1.0262)$ \\
\hline & I & 0.3167 & 0.3046 & 1.0396 & (0.9558-I.I308) \\
\hline & 2 & 0.0360 & 0.0379 & 0.9491 & $(0.7105-1.2679)$ \\
\hline \multirow[t]{3}{*}{ SNPIO } & 0 & 0.5933 & 0.6441 & 0.9211 & $(0.8805-0.9634)$ \\
\hline & 1 & 0.3712 & 0.3169 & 1.1713 & (1.0839-1.2657) \\
\hline & 2 & 0.0355 & 0.0389 & 0.9119 & $(0.6828-1.2180)$ \\
\hline \multirow[t]{3}{*}{ SNPII } & 0 & 0.6058 & 0.6142 & 0.9864 & $(0.9427-1.0321)$ \\
\hline & I & 0.3507 & 0.3352 & $|.046|$ & $(0.9675-1.1310)$ \\
\hline & 2 & 0.0435 & 0.0506 & 0.8601 & $(0.6650-1.1126)$ \\
\hline \multirow[t]{7}{*}{ (SNP9, SNPI0) } & 00 & 0.5863 & 0.6335 & 0.9255 & $(0.884 I-0.9689)$ \\
\hline & 01 & 0.0610 & 0.0240 & 2.5463 & $(1.9135-3.3885)$ \\
\hline & 10 & 0.0055 & 0.0107 & 0.5166 & $(0.2610-1.0224)$ \\
\hline & 11 & 0.3092 & 0.2919 & 1.0590 & $(0.97 \mid 7-1.1540)$ \\
\hline & 12 & 0.0020 & 0.0020 & 1.0018 & $(0.2831-3.5456)$ \\
\hline & 21 & 0.0010 & 0.0010 & 1.0018 & $(0.1676-5.9902)$ \\
\hline & 22 & 0.0335 & 0.0370 & 0.9071 & $(0.6734-1.2218)$ \\
\hline \multirow[t]{8}{*}{ (SNPI0, SNPII) } & 00 & 0.5463 & 0.5922 & 0.9224 & $(0.8776-0.9695)$ \\
\hline & 01 & 0.0455 & 0.0506 & 0.8997 & $(0.6982-1.1593)$ \\
\hline & 02 & 0.0015 & 0.0013 & I. $127 \mid$ & $(0.2525-5.0304)$ \\
\hline & 10 & 0.0595 & 0.0220 & 2.7095 & $(2.0164-3.6408)$ \\
\hline & 11 & 0.3032 & 0.2823 & 1.0739 & $(0.9839-1.1722)$ \\
\hline & 12 & 0.0085 & 0.0126 & 0.6723 & $(0.3805-1.1877)$ \\
\hline & 21 & 0.0020 & 0.0023 & 0.8587 & $(0.2517-2.9296)$ \\
\hline & 22 & 0.0335 & 0.0366 & 0.9153 & $(0.679 \mid-1.2336)$ \\
\hline
\end{tabular}

The explanation for how the relative risks are obtained and displayed is the same as that given in Table 3 . The major/minor alleles for rs6487236, rs I87। I 42 and rs 10770836 are A/G, G/A and G/A, respectively. The allelic information is extracted from the original T2D data. SNP9 = rs6487236, SNPIO = rs $187 I I 42$ and SNPII $=$ rs 10770836. 
Table 10: Haplotype relative risk evaluated from genotype distribution of SNPs in GYS2.

\begin{tabular}{|c|c|c|c|c|c|}
\hline \multirow[b]{2}{*}{ SNP } & \multirow[b]{2}{*}{ Allele and Haplotype } & \multicolumn{2}{|c|}{ Frequency } & \multirow[b]{2}{*}{ Relative Risk } & \multirow[b]{2}{*}{$95 \% \mathrm{Cl}$} \\
\hline & & Case & Control & & \\
\hline \multirow[t]{2}{*}{ SNP9 } & 0 & 0.8057 & 0.8098 & 0.9949 & $(0.9757-1.0146)$ \\
\hline & 1 & 0.1943 & 0.1902 & 1.0216 & $(0.9412-1.1087)$ \\
\hline \multirow[t]{2}{*}{ SNPIO } & 0 & 0.7789 & 0.8026 & 0.9705 & $(0.9505-0.9908)$ \\
\hline & 1 & 0.2211 & 0.1974 & 1.1201 & $(1.0367-1.2102)$ \\
\hline \multirow[t]{2}{*}{ SNPII } & 0 & 0.7811 & 0.7818 & 0.9992 & $(0.9782-1.0205)$ \\
\hline & 1 & 0.2189 & 0.2182 & 1.0030 & $(0.9299-1.0818)$ \\
\hline \multirow[t]{4}{*}{ (SNP9, SNPI0) } & 00 & 0.7740 & 0.7967 & 0.9715 & $(0.95 / 2-0.9922)$ \\
\hline & 01 & 0.0317 & 0.0131 & 2.4258 & $(1.8357-3.2056)$ \\
\hline & 10 & 0.0049 & 0.0059 & 0.8330 & $(0.4807-1.4433)$ \\
\hline & 11 & 0.1894 & 0.1843 & 1.0276 & $(0.9455-1.1169)$ \\
\hline \multirow[t]{4}{*}{ (SNPI0, SNPII) } & 00 & 0.7494 & 0.7692 & 0.9742 & $(0.9524-0.9965)$ \\
\hline & 01 & 0.0295 & 0.0334 & 0.8843 & $(0.7069-1.106 I)$ \\
\hline & 10 & 0.0318 & 0.0126 & 2.5275 & $(1.9064-3.35 I I)$ \\
\hline & 11 & 0.1894 & 0.1848 & 1.0244 & $(0.9426-1.1134)$ \\
\hline
\end{tabular}

The explanation for how the relative risks are obtained and displayed is the same as that given in Table 4 . The allelic information for each SNP is given in Table 9. SNP9 = rs6487236, SNPIO = rs I87II 42 and SNPII = rs 10770836.

culated from the contingency table of alleles or genotypes with disease status. On the other hand, a $\chi^{2}$ statistic for the deviation from HWE of each SNP in the case samples indicates the level of association. A large deviation from the equilibrium usually signifies strong association between a SNP and the disease. However, an excessively large deviation may be the result of genotyping errors. $n_{d}$ SNPs with largest test statistics for the deviation from HWE are hence excluded from the consideration.

The test statistics for the allelic association and deviation from HWE are multiplied together to form a single $S$ statistic for each remaining SNP. SNPs are then ranked according to their $S$ statistics. A preset number of SNPs with highest ranks are considered for association. The first candidate SNP set contains only the SNP with the highest rank (the highest $S$ statistic). The $p$-value for this first set is determined from a permutation simulation where the case and control labels are randomly permuted while the numbers of case and control samples remain unchanged. In each permutation replicate, a new genotype contingency table is constructed and a new $S$ statistic is subsequently obtained. The $p$-value is given by the fraction of permutation replicates with an $S$ statistic greater than or equal to the $S$ statistic from the original data. The second candidate SNP set consists of the first two SNPs in the rank list. The test statistic for this SNP set is the sum of $S$ statistics from both SNPs. The $p$-value for the second candidate SNP set is also obtained through the permutation simulation. By progressively adding the remaining SNP with the highest rank to the previously considered candidate set and performing the permutation simulation, $p$-values for all candidate SNP sets are estimated. The sizes of candidate SNP sets have the range of one to the preset number. Among all candidate sets, the SNP set that best describes genetic association has the lowest $p$-value.

Since multiple hypotheses are postulated during the construction of candidate SNP sets, the global $p$-value for the selected candidate set must be evaluated. This is achieved through a permutation simulation in which the current raw $p$-value for the chosen candidate set is now used as the test statistic. The existing permutation replicates, created for the early estimation of the raw $p$-value, can be reused and a nested permutation simulation is hence avoided. In this study, the maximum allowable size of the candidate

Table I I: Prediction accuracy of the best MDR model constructed from the 2LOmb output.

\begin{tabular}{lc}
\hline Description & Value \\
\hline SNP and Gene & rs226924I (PGMI), rs3790857 (PGMI), rs I89355I (PARK2), rs6924502 (PARK2), rs I87I I 42 (GYS2), \\
& rs I0770836 (GYS2) \\
Classification (Training) Accuracy & 0.5709 \\
Prediction Accuracy & 0.5402 \\
Cross-Validation Consistency (CVC) & $9 / 10$
\end{tabular}

The model contains six SNPs from PGMI, PARK2 and GYS2. A permutation test with I,000 randomised replicates of case-control data for this model reveals that the empirical $p$-value for the null hypothesis of no association is $p<0.001$. 
Table I2: Summary of prediction accuracy by MDR from early genetic association studies of T2D in a Korean population, a Han Chinese population from Taiwan, a female population from the US, and that from an early genetic association study of metabolic syndrome in an Italian population from the Centre East Coast Italy.

\begin{tabular}{lllccc}
\hline Reference & Population & Gene & Prediction Accuracy & CVC & Permutation p-value \\
\hline Cho et al. [43] & Korean & PPARG, UCP2 & 0.7957 & $9 / 10$ & 0.01 \\
Hsieh et al. [44] & Han Chinese & RXRG, EGFR & 0.6270 & $11 / 12$ & N/A \\
Qi et al. [45] & US & KCNJII, HNF4A & 0.5420 & $10 / 10$ & 0.010 \\
Fiorito et al. [47] & Italian & PPARG, DIO2 & 0.6170 & $10 / 10$ & 0.005
\end{tabular}

A permutation test with I,000 randomised replicates is performed to obtain the empirical $p$-value for the null hypothesis of no association in the studies conducted in the US and Italian populations. In contrast, a permutation test with 100 randomised replicates is performed to obtain the empirical $p$-value in the study conducted in the Korean population.

SNP set is the total number of available SNPs while the number of permutation replicates for $p$-value evaluation is set to 10,000 . The allelic association statistic employed in the study is the $\chi^{2}$ statistic that is obtained through the contingency table of genotypes with disease status. A PASCAL program for the set association approach can be obtained from the website for $S$ statistic in gene mapping [84].

\section{Correlation-based feature selection technique}

A correlation-based feature selection (CFS) technique [14] is an attribute (SNP) subset evaluation heuristic that considers both the usefulness of individual features (SNPs) in the (case-control) classification task and the level of intercorrelation among features. Each attribute subset is assigned a score given by

$$
\operatorname{Merit}_{F}=\frac{n_{c} \bar{r}_{c f}}{\sqrt{n_{c}+n_{c}\left(n_{c}-1\right) \bar{r}_{f f}}},
$$

where Merit $_{F}$ is the heuristic merit of an $n_{c}$-attribute subset $F, \bar{r}_{c f}$ is the average feature-class correlation and $\bar{r}_{f f}$ is the average feature-feature inter-correlation. An attribute subset receives a high merit score if it contains features that are highly correlated with the class and at the same time have low inter-correlation among one another. An application of a best-first search for the best subset identification is carried out to avoid searching through all possible attribute subsets. CFS has been integrated into a Weka package $[85,86]$.

\section{Tuned ReliefF}

A tuned ReliefF (TuRF) algorithm is a ranking algorithm for identifying genetic markers which are important in case-control classification [16]. TuRF is built on a ReliefF engine [15]. ReliefF randomly picks a sample from the (case-control) data and identifies its $n_{k}$ nearest neighbours from the same class and another $n_{k}$ nearest neighbours from the opposite class. The attribute values--the genotypes in this application--of the neighbour samples are compared to that of the randomly picked sample and are subsequently used to update the relevance score for each attribute (genetic marker). This process is repeated for a specified number of samples, which is limited by the total sample size. The rationale of ReliefF is that an attribute which is important for the classification should have different values for samples from different classes and have the same value for samples from the same class. The relevance score of an attribute have a range from -1 (not relevant) to +1 (highly relevant). TuRF exploits the capability of ReliefF by repeatedly executing ReliefF and removing a portion of worst attributes at the end of each execution. This leads to the reevaluation of remaining attributes and, hence, reduces the effects of attribute noise on the attribute screening. In this study, the number of repetitions for random sample picking in the ReliefF part is equal to the total number of case-control samples while the neighbourhood size $\left(n_{k}\right)$ for the relevance score calculation is set to ten. Furthermore, the worst $1 \%$ of SNPs is removed at the end of each ReliefF iteration (TuRF 1\%). TuRF has been integrated into the current distribution of multifactor dimensionality reduction (MDR) software.

Table 13: Penetrances for a two-locus interaction model.

\begin{tabular}{|c|c|c|c|c|}
\hline \multirow[b]{2}{*}{ Genotype } & \multicolumn{3}{|c|}{ Penetrance of Genotype } & \multirow[b]{2}{*}{ Marginal Penetrance } \\
\hline & $B B$ & Bb & $\boldsymbol{b} b$ & \\
\hline$A A$ & $f_{00}$ & $f_{01}$ & $f_{02}$ & $M_{A 0}$ \\
\hline$A a$ & $f_{10}$ & $f_{11}$ & $f_{12}$ & $M_{A I}$ \\
\hline$a a$ & $f_{20}$ & $f_{21}$ & $f_{22}$ & $M_{A 2}$ \\
\hline Marginal Penetrance & $M_{B 0}$ & $M_{B I}$ & $M_{B 2}$ & $K$ \\
\hline
\end{tabular}

$f_{i j}$ is the disease penetrance of genotype ij. $M_{A i}$ and $M_{B j}$ are the marginal penetrances for genotype $i$ at locus $A$ and genotype $j$ at locus $B$, respectively. $M_{A i}=M_{B j}=K, \forall i, j \in\{0, I, 2\}$ in a pure epistasis model. 
Table 14: Two-locus penetrances that lead to the maximum heritability $h_{\max }^{2}(K)=2 K /(I-K)$ for $K \in(0,1 / 4]$.

\begin{tabular}{lccc}
\hline \multirow{2}{*}{ Genotype } & \multicolumn{3}{c}{ Penetrance of Genotype } \\
& BB & Bb & b b \\
$A A$ & 0 & 0 & $4 K$ \\
$a a$ & 0 & $2 K$ & 0 \\
$a a$ & $4 K$ & 0 & 0
\end{tabular}

All allele frequencies are equal $\left(p_{A}=p_{B}=0.5\right)$.

\section{Multifactor dimensionality reduction}

A multifactor dimensionality reduction (MDR) method is a wrapper-based technique that is capable of identifying the best genetic marker combination among possible markers for the separation between case and control samples [19]. Similar to other wrapper-based methods, an $n_{f}$ fold cross-validation technique provides a means to determine the prediction accuracy of the candidate marker model. Basically, the combined case and control samples are randomly divided into $n_{f}$ folds where $n_{f}$ - 1 folds of samples are used to construct a decision table while the remaining fold of samples is used to identify the prediction capability of the constructed decision table. The decision table construction and testing procedure is repeated $n_{f}$ times. Hence, the samples in each fold are always used both to construct and to test the decision table. The number of cells in a decision table is given by $G^{n_{c}}$ where $n_{c}$ is the number of candidate markers selected from possible markers and $G$ is the number of possible genotypes according to the marker. For a SNP, which is a bi-allelic marker, $G$ is equal to three. During the decision table construction, each cell in the table is filled with case and control samples that have their genotype corresponds to the cell label. The ratio between numbers of case and control samples provides the decision for each cell whether the corresponding genotype is a protective or disease-predis-

Table 15: Three-locus penetrances that lead to the maximum heritability $h_{\max }^{2}(K)=9 K /(I-K)$ for $K \in(0,1 / 16]$.

\begin{tabular}{|c|c|c|c|c|c|c|c|c|c|}
\hline \multirow{3}{*}{ Genotype } & \multicolumn{9}{|c|}{ Penetrance of Genotype } \\
\hline & \multicolumn{3}{|c|}{$C C$} & \multicolumn{3}{|c|}{$C_{c}$} & \multicolumn{3}{|c|}{ cc } \\
\hline & BB & $\mathbf{B b}$ & $b \boldsymbol{b}$ & BB & $\mathbf{B b}$ & $b \boldsymbol{b}$ & BB & $\mathbf{B b}$ & $b b$ \\
\hline$A A$ & 0 & 0 & $16 K$ & 0 & 0 & 0 & 0 & 0 & 0 \\
\hline$A a$ & 0 & 0 & 0 & 0 & $4 K$ & 0 & 0 & 0 & 0 \\
\hline$a a$ & 0 & 0 & 0 & 0 & 0 & 0 & $16 K$ & 0 & 0 \\
\hline
\end{tabular}

All allele frequencies are equal $\left(p_{A}=p_{B}=p_{C}=0.5\right)$.
Table 16: Four-locus penetrances that lead to the maximum heritability $h_{\max }^{2}(K)=35 K /(I-K)$ for $K \in(0,1 / 64]$.

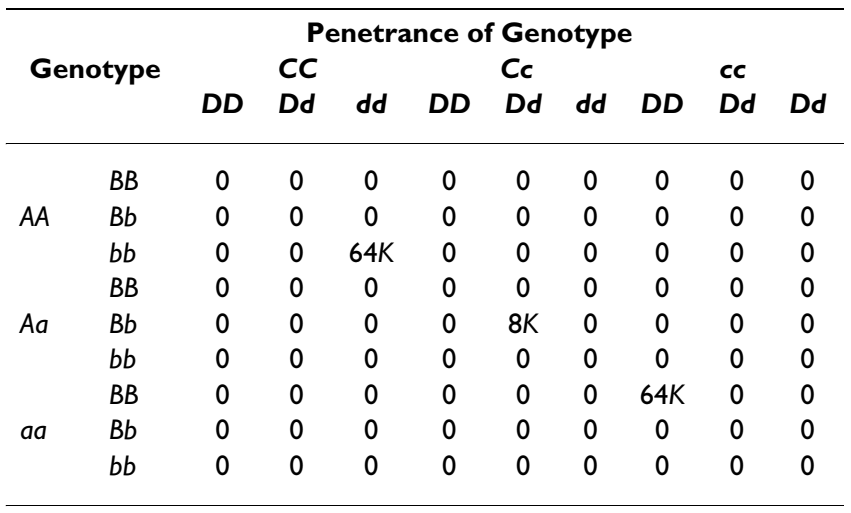

All allele frequencies are equal $\left(p_{A}=p_{B}=p_{C}=p_{D}=0.5\right)$.

posing genotype. An example of decision table construction is illustrated in Figure 13.

The prediction accuracy of the decision table is subsequently evaluated by counting the numbers of case and control samples in the testing fold that their disease status can correctly be identified using the constructed decision rules. The process of decision table construction and evaluation must be cycled through all or some of possible $2^{n_{m}}-1$ combinations where $n_{m}$ is the total number of available markers in the study. The best genetic marker combination is determined from two criteria: prediction accuracy and cross-validation consistency. Each time that a testing fold is used for the prediction accuracy determination, the accuracy of the interesting marker combination model is compared with that from other models that also contain the same number of markers. The model that consistently ranks the first in comparison to other choices with the same number of markers has high cross-validation consistency. Prediction accuracy is the main criterion for decision making while cross-validation consistency is only used as an auxiliary measure. Cross-validation consistency generally confirms that the high rank model can consistently be identified regardless of how the samples are divided for cross-validation. In a situation where two or more models with different number of markers are equally good in terms of prediction accuracy and crossvalidation consistency, the most parsimonious model-the combination with the least number of markers--is chosen as the best model.

After the best model has been selected, a permutation test is used to assess the probability of obtaining prediction accuracy that is at least as large as or larger than that observed in the original data from randomised data. This 
Table 17: Disease prevalence that gives the target maximum heritability of $0.01,0.025$ and 0.05 for two-, three- and four-locus interaction models.

\begin{tabular}{lccc}
\hline Model & & Prevalence (K) & $h_{\max }^{2}(K)=0.05$ \\
& $h_{\max }^{2}(K)=0.01$ & $h_{\max }^{2}(K)=0.025$ & 0.024390 \\
Two-locus & 0.004975 & 0.012346 & 0.005525 \\
Three-locus & 0.001110 & 0.002770 & 0.001427 \\
Four-locus & 0.000286 & 0.000714 & \\
\hline
\end{tabular}

represents the probability that the null hypothesis of no association is true. Each permutation replicate is constructed by randomly assigning the case/control status to each sample with the numbers of case and control samples remaining fixed. MDR analysis is subsequently carried out to obtain the prediction accuracy of each permutation replicate. The empirical $p$-value is denoted by the fraction of permutation replicates with the prediction accuracy greater than or equal to the prediction accuracy obtained from the original data. MDR software, which incorporates many additional features including interaction visualisation via dendrograms and genetic marker

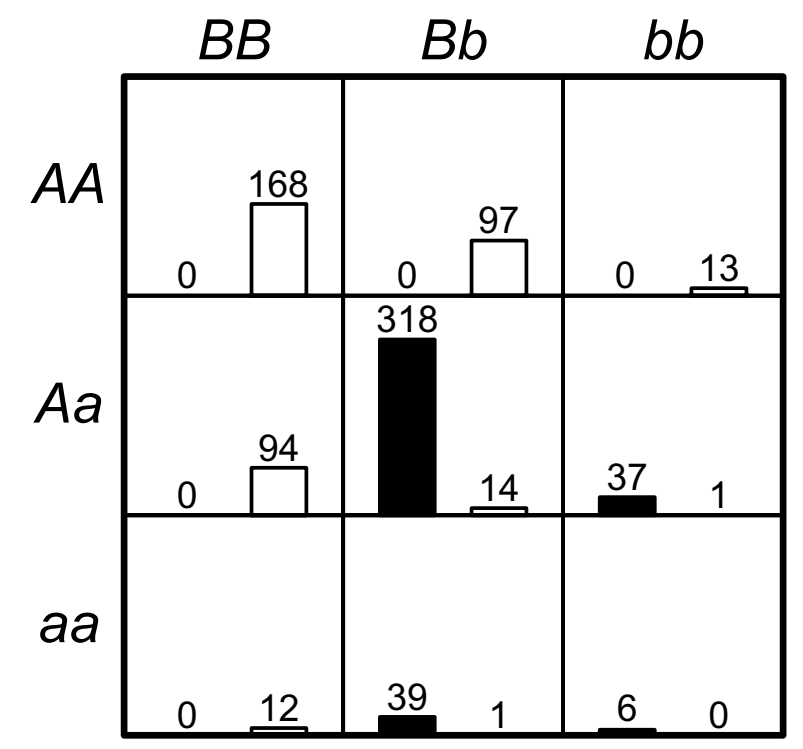

\section{Figure I 3}

An MDR decision table that is constructed using a balanced case-control data set with the sample size of $\mathbf{8 0 0}$. The genotype of each sample is determined from two SNPs. The table consists of nine cells where each cell represents a unique genotype. The left (black) bar in each cell represents the number of case samples while the right (white) bar represents the number of control samples. The cells with genotypes $A A B B, A A B b, A A b b, A a B B$ and $a a B B$ are labelled as protective genotypes while the cells with genotypes $A a B b, A a b b, a a B b$ and $a a b b$ are labelled as disease-predisposing genotypes. screening via a $\chi^{2}$ test, an odds ratio test, ReliefF and TuRF, is available from its homepage [87].

\section{JLIN}

JLIN or a Java LINkage disequilibrium plotter is a computer program for visualisation of linkage disequilibrium analysis [63]. The program is capable of displaying many statistical measures including $D^{\prime}[64]$ and $r^{2}$ [65]. The program is publicly available from the Centre for Genetic Epidemiology and Biostatistics, University of Western Australia [88].

\section{Interaction dendrogram}

An interaction dendrogram is a graphical tool for the visualisation of relationships among attributes (SNPs) $[68,69]$. The interaction dendrogram is constructed via hierarchical clustering analysis and is embedded into MDR software [87]. The dendrogram illustrates the entropy-based interaction between attributes by displaying interacting or related attributes closely together as adjacent leaves in a tree. At the same time, independent attributes are placed far apart from one another. In addition, the conclusion regarding whether the interaction between attributes is synergistic or redundancy is present can be deduced.

\section{Availability and requirements}

The 2LOmb program for Windows platforms and examples of simulated data are available at http:// code.google.com/p/nachol/w/list.

\section{List of abbreviations}

2LOmb: omnibus permutation test on ensembles of twolocus analyses; ALT: alanine transaminase; ANOVA: analysis of variance; AST: aspartate transaminase; CFS: correlation-based feature selection; $\mathrm{CI}$ : confidence interval; CVC: cross-validation consistency; DIO2: deiodinase, iodothyronine, type II; E2LA: exhaustive two-locus analysis; EGFR: epidermal growth factor receptor (erythroblastic leukemia viral (v-erb-b) oncogene homolog, avian); FAMHAP: software for single-marker analysis and joint analysis of unphased genotype data from tightly linked markers (haplotype analysis); FUSION: Finland-United States Investigation of NIDDM Genetics; genomeSIM: simulation package for generating case-control samples in large- 
scale and genome-wide association studies; GYS2: glycogen synthase 2 (liver); HNF4A: hepatocyte nuclear factor 4, alpha; HuGENet: Human Genome Epidemiology Network; HWE: Hardy-Weinberg equilibrium; JLIN: Java LINkage disequilibrium plotter; KCNJ11: potassium inwardly-rectifying channel, subfamily J, member 11; LD: linkage disequilibrium; LIM domains: protein structural domains that are named after their initial discovery in the proteins Lin11, Isl-1 and Mec-3; LMX1A: LIM homeobox transcription factor 1, alpha; MDR: multifactor dimensionality reduction; NIDDM: noninsulin-dependent diabetes mellitus; PARK2: Parkinson disease (autosomal recessive, juvenile) 2, parkin; PGM1: phosphoglucomutase 1; PPARG: peroxisome proliferator-activated receptor gamma; $R X R G$ : retinoid $\mathrm{X}$ receptor, gamma; $\mathrm{SAA}$ : set association approach; SNP: single nucleotide polymorphism; T2D: type 2 diabetes mellitus; TuRF: tuned ReliefF; UCP2: uncoupling protein 2 (mitochondrial, proton carrier); Weka: Waikato environment for knowledge analysis; WTCCC: Wellcome Trust Case Control Consortium.

\section{Authors' contributions}

WW conducted the literature survey, formulated the research question, implemented the proposed algorithm, designed the experiment, and collected and interpreted the computational results. AA conducted the literature survey, formulated the research question, designed the experiment and secured the access to the genomeSIM package. TP performed the statistical analysis and interpreted the statistical results. SS monitored and oversaw the execution of computer programs on the Beowulf cluster. CL provided additional comments about the genetic association study of T2D. NC conducted the literature survey, formulated the research question, designed the proposed algorithm, designed the experiment, secured the access to the T2D data from WTCCC, selected the candidate genes for the T2D association study, discussed all results, drew the conclusions and wrote the manuscript. All authors read and approved the final manuscript.

\section{Authors' information}

WW is a Ph.D. student at the Department of Electrical Engineering, Faculty of Engineering, King Mongkut's University of Technology North Bangkok. He also received his B.Eng. and M.Eng. degrees in electrical engineering from King Mongkut's University of Technology North Bangkok. His current research interests include machine learning, evolutionary computation and bioinformatics.

AA is a Ph.D. student at the Department of Immunology, Faculty of Medicine Siriraj Hospital, Mahidol University. He also received his B.Sc. degree in pharmacy from Mahidol University. His current research interests include human genetics, genetic epidemiology, population genetics and bioinformatics.
TP is a Ph.D. student at the Department of Electrical Engineering, Faculty of Engineering, King Mongkut's University of Technology North Bangkok. He also received his B.Eng. and M.Eng. degrees in production engineering from King Mongkut's University of Technology North Bangkok. His current research interests include evolutionary multi-objective optimisation and machine learning.

SS is a part-time research assistant at the Department of Electrical Engineering, Faculty of Engineering, King Mongkut's University of Technology North Bangkok. He received his B.Eng. and M.Eng. degrees in electrical engineering from Thammasat University and King Mongkut's University of Technology North Bangkok, respectively. His current research interests include machine learning and genetic epidemiology.

CL is the Head of Division of Molecular Genetics at the Department of Research and Development, Faculty of Medicine Siriraj Hospital, Mahidol University. He also received his M.D. degree from Mahidol University. His current research interests include human genetics and genetic diseases.

$\mathrm{NC}$ is an associate professor of electrical engineering at King Mongkut's University of Technology North Bangkok and an adjunct professor of genetic epidemiology at Mahidol University. He received his B.Eng. and Ph.D. degrees from the Department of Automatic Control and Systems Engineering, University of Sheffield. His current research interests include evolutionary computation, machine learning and genetic epidemiology.

\section{Additional material}

\section{Additional file 1}

List of SNPs for the association study of T2D. This Excel spreadsheet file contains the information about 7,065 SNPs which are explored during the genetic association study of T2D. Bonferroni-corrected and uncorrected $\chi^{2}$ 's $\mathrm{p}$-values from single-locus analyses are also provided.

Click here for file

[http://www.biomedcentral.com/content/supplementary/14712105-10-294-S1.xls]

\section{Additional file 2}

genomeSIM parameters. This text file contains an example of parameter setting in the genomeSIM simulation package.

Click here for file

[http://www.biomedcentral.com/content/supplementary/14712105-10-294-S2.txt]

\section{Acknowledgements}

The authors are extremely grateful to four anonymous reviewers and Dr. Danielle Talbot for their valuable comments and suggestions, which have contributed a lot towards improving the content and presentation of this 
article. The authors are also extremely grateful to Dr. Kuntinee Maneeratana, Dr. Pensiri Tongpadungrod, Dr. Graeme James Sheppard and Mr. Edward L. Karas for their efforts on proofreading the manuscript. The authors wish to thank Dr. Marong Phadoongsidhi and Dr. Vara Varavithya and for their assistance on processing the T2D data and providing an access to the Beowulf cluster, respectively. The authors also wish to thank Dr. Sissades Tongsima for his assistance on testing of multiple hypotheses, providing many suggestions for the manuscript revision and allowing the usage of computer facilities at the Genome Institute, National Center for Genetic Engineering and Biotechnology (BIOTEC), National Science and Technology Development Agency (NSTDA) during the algorithm development. The authors acknowledge Scott M. Dudek at the Vanderbilt University for providing an access to the genomeSIM package. Furthermore, the authors acknowledge Dr. Audrey Duncanson and the Wellcome Trust Case Control Consortium for granting an access to the genome-wide case-control data. WW, AA and TP were supported by the Thailand Research Fund (TRF) through the Royal Golden Jubilee Ph.D. Programme (Grant No. PHD/I.E.KN.49/A.I, PHD/4.I.MU.45/C.I and PHD/I.E.KN.50/A.I, respectively). CL was supported by the Mahidol Research Grant. NC was supported by the Thailand Research Fund and the National Science and Technology Development Agency through the NSTDA Chair Professor Grant.

\section{References}

I. Risch N, Merikangas K: The future of genetic studies of complex human diseases. Science 1996, 273:1516-1517.

2. Musani SK, Shriner D, Liu N, Feng R, Coffey CS, Yi N, Tiwari HK, Allison $\mathrm{DB}$ : Detection of gene $\times$ gene interactions in genomewide association studies of human population data. Hum Hered 2007, 63:67-84.

3. The Wellcome Trust Case Control Consortium: Genome-wide association study of 14,000 cases of seven common diseases and 3,000 shared controls. Nature 2007, 447:661-678.

4. The GAIN Collaborative Research Group: New models of collaboration in genome-wide association studies: the Genetic Association Information Network. Nat Genet 2007, 39:|045-|05|.

5. Heidema AG, Boer JMA, Nagelkerke N, Mariman ECM, van der A DL, Feskens EJM: The challenge for genetic epidemiologists: how to analyze large numbers of SNPs in relation to complex diseases. BMC Genet 2006, 7:23.

6. Motsinger AA, Ritchie MD, Reif DM: Novel methods for detecting epistasis in pharmacogenomics studies. Pharmacogenomics 2007, 8: I229-I24I.

7. Moore JH, Gilbert JC, Tsai CT, Chiang FT, Holden T, Barney N, White BC: A flexible computational framework for detecting, characterizing, and interpreting statistical patterns of epistasis in genetic studies of human disease susceptibility. J Theor Biol 2006, 24I:252-26I.

8. Saeys Y, Inza I, Larrañaga P: A review of feature selection techniques in bioinformatics. Bioinformatics 2007, 23:2507-25I7.

9. Lewis CM: Genetic association studies: design, analysis and interpretation. Brief Bioinform 2002, 3:146-153.

10. Montana G: Statistical methods in genetics. Brief Bioinform 2006, 7:297-308

II. Hoh J, Wille A, Ott J: Trimming, weighting, and grouping SNPs in human case-control association studies. Genome Res 200 I, I I:2 | | 5-2 | 19

12. Potter DM: Omnibus permutation tests of the association of an ensemble of genetic markers with disease in case-control studies. Genet Epidemiol 2006, 30:438-446.

13. Chapman J, Clayton D: Detecting association using epistatic information. Genet Epidemiol 2007, 3 1:894-909.

14. Hall MA, Holmes G: Benchmarking attribute selection techniques for discrete class data mining. IEEE Trans Knowl Data Eng 2003, I 5: | 437-| 447.

15. Robnik-Šikonja M, Kononenko I: Theoretical and empirical analysis of ReliefF and RReliefF. Mach Learn 2003, 53:23-69.

16. Moore JH, White BC: Tuning ReliefF for genome-wide genetic analysis. In Evolutionary Computation, Machine Learning and Data Mining in Bioinformatics Edited by: Marchiori E, Moore JH, Rajapakse JC.
Berlin, Heidelberg: Springer; 2007:166-175. [Goos G, Hartmanis ], van Leeuwen J (Founding and Former Series Editors): Lecture Notes in Computer Science, vol 4447].

17. Nelson MR, Kardia SLR, Ferrell RE, Sing CF: A combinatorial partitioning method to identify multilocus genotypic partitions that predict quantitative trait variation. Genome Res 200I, I I:458-470.

18. Culverhouse R, Klein T, Shannon W: Detecting epistatic interactions contributing to quantitative traits. Genet Epidemiol 2004, 27: $|4|-152$

19. Ritchie MD, Hahn LW, Roodi N, Bailey LR, Dupont WD, Parl FF, Moore $\mathrm{JH}$ : Multifactor-dimensionality reduction reveals highorder interactions among estrogen-metabolism genes in sporadic breast cancer. Am J Hum Genet 2001, 69:138-I47.

20. Hahn LW, Ritchie MD, Moore JH: Multifactor dimensionality reduction software for detecting gene-gene and gene-environment interactions. Bioinformatics 2003, 19:376-382.

21. Bush WS, Dudek SM, Ritchie MD: Parallel multifactor dimensionality reduction: a tool for the large-scale analysis of genegene interactions. Bioinformatics 2006, 22:2173-2174.

22. Chung Y, Lee SY, Elston RC, Park T: Odds ratio based multifactor-dimensionality reduction method for detecting genegene interactions. Bioinformatics 2007, 23:71-76.

23. Bush WS, Edwards TL, Dudek SM, McKinney BA, Ritchie MD: Alternative contingency table measures improve the power and detection of multifactor dimensionality reduction. BMC Bioinformatics 2008, 9:238.

24. Lou XY, Chen GB, Yan L, Ma JZ, Mangold JE, Zhu J, Elston RC, Li MD: A combinatorial approach to detecting gene-gene and geneenvironment interactions in family studies. Am J Hum Genet 2008, 83:457-467.

25. Edwards TL, Lewis K, Velez DR, Dudek SM, Ritchie MD: Exploring the performance of multifactor dimensionality reduction in large scale SNP studies and in the presence of genetic heterogeneity among epistatic disease models. Hum Hered 2009, 67:183-192.

26. Mechanic LE, Luke BT, Goodman JE, Chanock SJ, Harris CC: Polymorphism Interaction Analysis (PIA): a method for investigating complex gene-gene interactions. BMC Bioinformatics 2008, 9: 146.

27. Liang KH, Hwang Y, Shao WC, Chen EY: An algorithm for model construction and its applications to pharmacogenomic studies. J Hum Genet 2006, 5 I:751-759.

28. Estrada-Gil JK, Fernández-López JC, Hernández-Lemus E, SilvaZolezzi I, Hidalgo-Miranda A, Jiménez-Sánchez G, Vallejo-Clemente EE: GPDTI: a Genetic Programming Decision Tree Induction method to find epistatic effects in common complex diseases. Bioinformatics 2007, 23:il67-il74.

29. Nunkesser R, Bernholt T, Schwender H, Ickstadt K, Wegener I: Detecting high-order interactions of single nucleotide polymorphisms using genetic programming. Bioinformatics 2007, 23:3280-3288.

30. Lunetta KL, Hayward LB, Segal J, van Eerdewegh P: Screening largescale association study data: exploiting interactions using random forests. BMC Genet 2004, 5:32.

31. Bureau A, Dupuis J, Falls K, Lunetta KL, Hayward B, Keith TP, van Eerdewegh P: Identifying SNPs predictive of phenotype using random forests. Genet Epidemiol 2005, 28: I71-182.

32. Chen X, Liu CT, Zhang $M$, Zhang H: A forest-based approach to identifying gene and gene-gene interactions. Proc Natl Acad Sci USA 2007, 104:19199-19203.

33. Ritchie MD, White BC, Parker JS, Hahn LW, Moore JH: Optimization of neural network architecture using genetic programming improves detection and modeling of gene-gene interactions in studies of human diseases. BMC Bioinformatics 2003, 4:28.

34. Motsinger-Reif AA, Dudek SM, Hahn LW, Ritchie MD: Comparison of approaches for machine-learning optimization of neural networks for detecting gene-gene interactions in genetic epidemiology. Genet Epidemiol 2008, 32:325-340.

35. Cordell HJ: Epistasis: what it means, what it doesn't mean, and statistical methods to detect it in humans. Hum Mol Genet 2002, I I :2463-2468.

36. Wilson SR: Epistasis. In Nature Encyclopedia of the Human Genome Volume 2. Edited by: Cooper DN. London: Nature Publishing Group; 2004:3 I 7-320. 
37. Neuman RJ, Rice JP: Two-locus models of disease. Genet Epidemiol 1992, 9:347-365.

38. Schork NJ, Boehnke M, Terwilliger JD, Ott J: Two-trait-locus linkage analysis: a powerful strategy for mapping complex genetic traits. Am J Hum Genet 1993, 53: I I27-I I36.

39. Li W, Reich J: A complete enumeration and classification of two-locus disease models. Hum Hered 2000, 50:334-349.

40. Marchini J, Donnelly P, Cardon LR: Genome-wide strategies for detecting multiple loci that influence complex diseases. Nat Genet 2005, 37:413-417.

41. Hallgrímsdóttir IB, Yuster DS: A complete classification of epistatic two-locus models. BMC Genet 2008, 9:17.

42. Culverhouse R, Suarez BK, Lin J, Reich T: A perspective on epistasis: limits of models displaying no main effect. Am J Hum Genet 2002, 70:46|-47|

43. Cho YM, Ritchie MD, Moore JH, Park JY, Lee KU, Shin HD, Lee HK, Park KS: Multifactor-dimensionality reduction shows a twolocus interaction associated with type 2 diabetes mellitus. Diabetologia 2004, 47:549-554.

44. Hsieh CH, Liang KH, Hung Y], Huang LC, Pei D, Liao YT, Kuo SW, Bey MSJ, Chen JL, Chen EY: Analysis of epistasis for diabetic nephropathy among type 2 diabetic patients. Hum Mol Genet 2006, I 5:270|-2708.

45. Qi L, van Dam RM, Asselbergs FW, Hu FB: Gene-gene interactions between HNF4A and KCN $I I$ in predicting type 2 diabetes in women. Diabet Med 2007, 24: I I87-II9I.

46. Zhang Z, Zhang S, Wong MY, Wareham NJ, Sha Q: An ensemble learning approach jointly modeling main and interaction effects in genetic association studies. Genet Epidemiol 2008 32:285-300.

47. Fiorito M, Torrente I, De Cosmo S, Guida V, Colosimo A, Prudente S, Flex E, Menghini R, Miccoli R, Penno G, Pellegrini F, Tassi V, Federici $M$, Trischitta V, Dallapiccola B: Interaction of DIO2 T92A and PPAR 2 PI2A polymorphisms in the modulation of metabolic syndrome. Obesity 2007, I5:2889-2895.

48. Albrechtsen A, Castella S, Andersen G, Hansen T, Pedersen $O$ Nielsen R: A Bayesian multilocus association method: allowing for higher-order interaction in association studies. Genetics 2007, 176: I 197-I 208.

49. Zhang Y, Liu JS: Bayesian inference of epistatic interactions in case-control studies. Nat Genet 2007, 39: I 167-I I73.

50. Evans DM, Marchini J, Morris AP, Cardon LR: Two-stage two-locus models in genome-wide association. PLoS Genet 2006, 2:eI57.

5I. Ionita I, Man M: Optimal two-stage strategy for detecting interacting genes in complex diseases. BMC Genet 2006, 7:39.

52. Gayán J, González-Pérez A, Bermudo F, Sáez ME, Royo JL, Quintas A, Galan J], Morón FJ, Ramirez-Lorca R, Real LM, Ruiz A: A method for detecting epistasis in genome-wide studies using case-control multi-locus association analysis. BMC Genomics 2008, 9:360.

53. Heidema AG, Feskens EJM, Doevendans PAFM, Ruven HJT, van Houwelingen HC, Mariman ECM, Boer JMA: Analysis of multiple SNPs in genetic association studies: comparison of three multilocus methods to prioritize and select SNPs. Genet Epidemiol 2007, $31: 910-921$.

54. Pesarin F: Multivariate Permutation Tests with Applications to Biostatistics Chichester: Wiley; 200I.

55. Fisher RA: Statistical Methods for Research Workers 4th edition. London: Oliver and Boyd; 1932

56. Westfall PH, Young SS: Resampling-Based Multiple Testing: Examples and Methods for p-value Adjustment New York: John Wiley and Sons; 1993.

57. Becker T, Schumacher J, Cichon S, Baur MP, Knapp M: Haplotype interaction analysis of unlinked regions. Genet Epidemiol 2005, 29:313-322

58. Herold C, Becker T: Genetic association analysis with FAMHAP: a major program update. Bioinformatics 2009, 25: I34-136.

59. Hardy $\mathrm{GH}$ : Mendelian proportions in a mixed population. Science 1908, 28:49-50.

60. Dudek SM, Motsinger AA, Velez DR, Williams SM, Ritchie MD: Data simulation software for whole-genome association and other studies in human genetics. In Proceedings of the Pacific Symposium on Biocomputing 2006: 3-7 January 2006; Maui Edited by: Altman RB Dunker AK, Hunter L, Murray T, Klein TE. Singapore: World Scientific; 2006:499-5I0.

61. Guyon I, Elisseeff A: An introduction to variable and feature selection. J Mach Learn Res 2003, 3: I I57-I I82.
62. Yu W, Gwinn M, Clyne M, Yesupriya A, Khoury MJ: A navigator for human genome epidemiology. Nat Genet 2008, 40: I24- 125.

63. Carter KW, McCaskie PA, Palmer LJ: JLIN: a java based linkage disequilibrium plotter. BMC Bioinformatics 2006, 7:60.

64. Lewontin RC: The interaction of selection and linkage. I. general considerations; heterotic models. Genetics 1964, 49:49-67.

65. Hill WG, Robertson A: Linkage disequilibrium in finite populations. Theor Appl Genet 1968, 38:226-231.

66. Excoffier L, Slatkin M: Maximum-likelihood estimation of molecular haplotype frequencies in a diploid population. Mol Biol Evol 1995, 1 2:92 I-927.

67. Epstein MP, Satten GA: Inference on haplotype effects in casecontrol studies using unphased genotype data. Am J Hum Genet 2003, 73:1316-1329.

68. Jakulin A, Bratko I, Smrke D, Demšar J, Zupan B: Attribute interactions in medical data analysis. In Artificial Intelligence in Medicine Edited by: Dojat M, Keravnou E, Barahona P. Berlin, Heidelberg: Springer; 2003:229-238. [Carbonell JG, Siekmann J (Series Editors): Lecture Notes in Artificial Intelligence, vol 2780].

69. Jakulin A, Bratko I: Analyzing attribute dependencies. In Knowledge Discovery in Databases: PKDD 2003 Edited by: Lavrač N, Gamberger D, Todorovski L, Blockeel $\mathrm{H}$. Berlin, Heidelberg: Springer; 2003:229-240. [Carbonell JG, Siekmann J (Series Editors): Lecture Notes in Artificial Intelligence, vol 2838].

70. Kanehisa M, Goto S: KEGG: Kyoto Encyclopedia of Genes and Genomes. Nucleic Acids Res 2000, 28:27-30.

7I. Kanehisa M, Goto S, Hattori M, Aoki-Kinoshita K, Itoh M, Kawashima S, Katayama T, Araki M, Hirakawa M: From genomics to chemical genomics: new developments in KEGG. Nucleic Acids Res 2006, 34:D354-D357.

72. Kanehisa M, Araki M, Goto S, Hattori M, Hirakawa M, Itoh M, Katayama T, Kawashima S, Okuda S, Tokimatsu T, Yamanishi $Y$ : KEGG for linking genomes to life and the environment. Nucleic Acids Res 2008, 36:D480-D484.

73. Thameem F, Wolford JK, Wang J, German MS, Bogardus C, Prochazka M: Cloning, expression and genomic structure of human LMXIA, and variant screening in Pima Indians. Gene 2002, 290:217-225.

74. Hanson RL, Ehm MG, Pettitt DJ, Prochazka M, Thompson DB, Timberlake D, Foroud T, Kobes S, Baier L, Burns DK, Almasy L, Blangero J, Garvey WT, Bennett PH, Knowler WC: An autosomal genomic scan for loci linked to type II diabetes mellitus and bodymass index in Pima Indians. Am J Hum Genet 1998, 63: I I30- I I 38

75. Leak TS, Mychaleckyj JC, Smith SG, Keene KL, Gordon CJ, Hicks PJ, Freedman BI, Bowden DW, Sale MM: Evaluation of a SNP map of 6q24-27 confirms diabetic nephropathy loci and identifies novel associations in type 2 diabetes patients with nephropathy from an African-American population. Hum Genet 2008, |24:63-7|.

76. Sale MM, Freedman $\mathrm{BI}$, Langefeld $\mathrm{CD}$, Williams $\mathrm{AH}$, Hicks PJ, Colicigno CJ, Beck SR, Brown WM, Rich SS, Bowden DW: A genomewide scan for type 2 diabetes in African-American families reveals evidence for a locus on chromosome 6q. Diabetes 2004, 53:830-837.

77. Watanabe I, Tomita A, Shimizu M, Sugawara M, Yasumo H, Koishi R, Takahashi T, Miyoshi K, Nakamura K, Izumi T, Matsushita Y, Furukawa $H$, Haruyama $H$, Koga T: A study to survey susceptible genetic factors responsible for troglitazone-associated hepatotoxicity in Japanese patients with type 2 diabetes mellitus. Clin Pharmacol Ther 2003, 73:435-455.

78. Gloria-Bottini F, Magrini A, Antonacci E, La Torre M, Di Renzo L, De Lorenzo A, Bergamaschi A, Bottini E: Phosphoglucomutase genetic polymorphism and body mass. Am J Med Sci 2007, 334:42I-425.

79. Spencer N, Hopkinson DA, Harris H: Phosphoglucomutase polymorphism in man. Nature 1964, 204:742-745.

80. March RE, Putt W, Hollyoake M, Ives JH, Lovegrove JU, Hopkinson DA, Edwards YH, Whitehouse DB: The classical human phosphoglucomutase (PGMI) isozyme polymorphism is generated by intragenic recombination. Proc Natl Acad Sci USA 1993. 90:10730-10733.

8I. Zeggini E, Scott LJ, Saxena R, Voight BF: Meta-analysis of genomewide association data and large-scale replication identifies additional susceptibility loci for type 2 diabetes. Nat Genet 2008, 40:638-645. 
82. Press WH, Teukolsky SA, Vetterling WT, Flannery BP: Numerical Recipes in C: The Art of Scientific Computing 2nd edition. Cambridge: Cambridge University Press; 1992.

83. Moore JH, Hahn LW, Ritchie MD, Thornton TA, White BC: Routine discovery of complex genetic models using genetic algorithms. Appl Soft Comput 2004, 4:79-86.

84. S Statistic in Gene Mapping [http://www.genemapping.cn/sum stat.html]

85. Witten IH, Frank E: Data Mining: Practical Machine Learning Tools and Techniques 2nd edition. San Francisco: Morgan Kaufmann; 2005.

86. Weka 3: Data Mining Software in Java [http:// www.cs.waikato.ac.nz/ml/weka/]

87. Multifactor Dimensionality Reduction [http://www.multifac tordimensionalityreduction.org/]

88. JLIN: A Java Based Linkage Disequilibrium Plotter [http:// www.genepi.org.au/jin.html]

Publish with Bio Med Central and every scientist can read your work free of charge

"BioMed Central will be the most significant development for disseminating the results of biomedical research in our lifetime. "

Sir Paul Nurse, Cancer Research UK

Your research papers will be:

- available free of charge to the entire biomedical community

- peer reviewed and published immediately upon acceptance

- cited in PubMed and archived on PubMed Central

- yours - you keep the copyright

Submit your manuscript here:

http://www.biomedcentral.com/info/publishing_adv.asp
BioMedcentral 